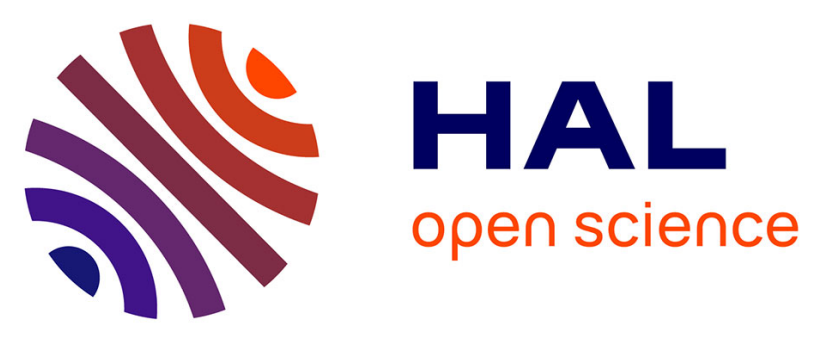

\title{
Comparison of conventional and high-sensitivity troponin in patients with chest pain: A collaborative meta-analysis
}

Michael Lipinski, Nevin Baker, Ricardo Escárcega, Rebecca Torguson, Fang Chen, Sally Aldous, Michael Christ, Paul Collinson, Steve Goodacre, Johannes Mair, et al.

\section{To cite this version:}

Michael Lipinski, Nevin Baker, Ricardo Escárcega, Rebecca Torguson, Fang Chen, et al.. Comparison of conventional and high-sensitivity troponin in patients with chest pain: A collaborative meta-analysis. American Heart Journal, 2015, 169 (1), pp.6 - 16.e6. 10.1016/j.ahj.2014.10.007 . hal-01768312

\section{HAL Id: hal-01768312 \\ https://hal.umontpellier.fr/hal-01768312}

Submitted on 3 Feb 2020

HAL is a multi-disciplinary open access archive for the deposit and dissemination of scientific research documents, whether they are published or not. The documents may come from teaching and research institutions in France or abroad, or from public or private research centers.
L'archive ouverte pluridisciplinaire HAL, est destinée au dépôt et à la diffusion de documents scientifiques de niveau recherche, publiés ou non, émanant des établissements d'enseignement et de recherche français ou étrangers, des laboratoires publics ou privés. 


\section{Comparison of conventional and}

\section{high-sensitivity troponin in patients with chest pain: A collaborative meta-analysis}

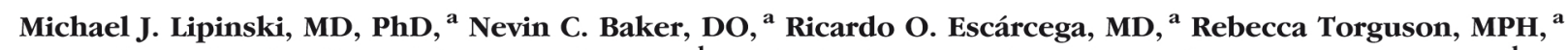
Fang Chen, PhD, ${ }^{\mathrm{a}}$ Sally J. Aldous, MBChB, MD, ${ }^{\mathrm{b}}$ Michael Christ, MD, ${ }^{\mathrm{c}}$ Paul O. Collinson, MD, FRCPath, ${ }^{\mathrm{d}}$ Steve W. Goodacre, PhD, ${ }^{\mathrm{e}}$ Johannes Mair, MD, ${ }^{\mathrm{f}}$ Kenji Inoue, MD, PhD, ${ }^{\mathrm{g}}$ Ulrich Lotze, MD,${ }^{\mathrm{h}}$

Mustapha Sebbane, MD, PhD, ${ }^{\mathrm{i}}$ Jean-Paul Cristol, $\mathrm{MD}, \mathrm{PhD},{ }^{\mathrm{j}}$ Yonathan Freund, $\mathrm{MD},{ }^{\mathrm{k}}$

Camille Chenevier-Gobeaux, PharmD, PhD, ${ }^{1}$ Christophe Meune, MD, PhD, ${ }^{\mathrm{m}, \mathrm{n}}$ Kai M. Eggers, MD, PhD, ${ }^{\circ}$ Radosław Pracoń, MD, ${ }^{\mathbf{p}}$ Donald H. Schreiber, MD, ${ }^{\mathrm{q}}$ Alan H. B. Wu, PhD, ${ }^{\mathrm{r}}$ Jordi Ordoñez-Llanos, MD, PhD, ${ }^{\text {s,t }}$ Allan S. Jaffe, MD, ${ }^{\mathrm{u}, \mathrm{v}}$ Raphael Twerenbold, MD, ${ }^{\mathrm{w}, \mathrm{x}}$ Christian Mueller, MD, ${ }^{\mathrm{w}}$ and Ron Waksman, MD $^{\mathrm{a}}$ Washington, DC; Christchurch, New Zealand; Nuremberg, Frankenbausen, Bad Krozingen, Germany; London, Sheffield, United Kingdom; Innsbruck, Austria; Tokyo, Japan; Montpellier, Paris, Bobigny, France; Uppsala, Sweden; Warsaw, Poland; Stanford, San Francisco, CA; Barcelona, Spain; Rochester, MN; and Basel, Switzerland

Background Multiple studies have evaluated the diagnostic and prognostic performance of conventional troponin (cTn) and high-sensitivity troponin (hs-cTn). We performed a collaborative meta-analysis comparing cTn and hs-cTn for diagnosis of acute myocardial infarction (AMI) and assessment of prognosis in patients with chest pain.

Methods MEDLINE/PubMed, Cochrane CENTRAL, and EMBASE were searched for studies assessing both cTn and hscTn in patients with chest pain. Study authors were contacted and many provided previously unpublished data.

Results From 17 included studies, there were 8,644 patients. Compared with baseline cTn, baseline hs-cTn had significantly greater sensitivity $(0.884$ vs $0.749, P<.001)$ and negative predictive value (NPV; 0.964 vs $0.935, P<.001)$, whereas specificity $(0.816$ vs $0.938, P<.001)$ and positive predictive value $(0.558$ vs $0.759, P<.001)$ were significantly reduced. Based on summary receiver operating characteristic curves, test performance for the diagnosis of AMI was not significantly different between baseline cTn and hs-cTn $(0.90[95 \% \mathrm{Cl} 0.85-0.95]$ vs $0.92[95 \% \mathrm{Cl} 0.90-0.94])$. In a subanalysis of 6 studies that alternatively defined AMI based on hs-cTn, cTn had lower sensitivity $(0.666, P<.001)$ and NPV (0.906, $P<.001)$. Elevation of baseline hs-cTn, but negative baseline cTn, was associated with increased risk of death or nonfatal myocardial infarction during follow-up $(P<.001)$ compared with both negative.

Conclusion High-sensitivity troponin has significantly greater early sensitivity and NPV for the diagnosis of AMI at the cost of specificity and positive predictive value, which may enable early rule in/out of AMl in patients with chest pain. Baseline hs-cTn elevation in the setting of negative cTn is also associated with increased nonfatal myocardial infarction or death during follow-up.

From the aMedStar Cardiovascular Research Network, MedStar Heart Institute, Medstar Washington Hospital Center, Washington, DC, bDepartment of Cardiology, Christchurch Hospital, Christchurch, New Zealand, 'Department of Emergency and Critical Care Medicine, Paracelsus Medical University, Nuremberg, Germany, ${ }^{d}$ Clinical Blood Sciences Laboratory, St George's Hospital, London, United Kingdom, '${ }^{\circ}$ School of Health and Related Research, University of Sheffield, Sheffield, United Kingdom, 'Department of Internal Medicine, Innsbruck Medical University, Innsbruck, Austria, ${ }^{9}$ Department of Cardiology, Juntendo University Nerima Hospital, Tokyo, Japan, 'Department of Internal Medicine, DRK-Manniske-Krankenhaus Bad Frankenhausen, Frankenhausen, Germany, 'Départment des urgences, Centre Hospitalier Régional Universitaire Lapeyronie, Montpellier, France, 'Département de Biochimie, Centre Hospitalier Régional Universitaire Lapeyronie, Montpellier, France, ${ }^{k}$ Department of Emergency Medicine and Surgery, Hôspital PitiéSalpétrière, APHP, Université Pierre et Marie Curie-Paris 6 (UPMC), Paris, France, 'Clinical Chemistry Laboratory, Groupe Hospitalier Cochin-Broca-Hôtel Dieu, Université Paris Descartes, Assistance Publique des Hôpitaux de Paris, Paris, France, ${ }^{m}$ Cardiology Department, Groupe Hospitalier Cochin-Broca-Hôtel Dieu, Université Paris Descartes, Assistance Publique des Hôpitaux de Paris, Paris, France, " Cardiology Department, Paris XIII University, Avicenne Hospital, Bobigny, France, 'Department of Medical Sciences,

\footnotetext{
Uppsala University, Uppsala, Sweden, ${ }^{P}$ Department of Coronary and Structural Heart Diseases, National Insititute of Cardiology, Warsaw, Poland, ${ }^{9}$ Division of Emergency Medicine, Stanford University School of Medicine, Stanford, CA, 'Department of Laboratory Medicine, University of California, San Francisco, CA, ${ }^{s}$ Servei de Bioquímica Clínica, Hospital de Sant Pau, Barcelona, Spain, 'Biochemistry and Molecular Biology Department, Universitat Autònoma, Barcelona, Spain, "Division of Cardiology, Mayo Clinic College of Medicine, Rochester, MN, 'Department of Laboratory Medicine and Pathology, Mayo Clinic College of Medicine, Rochester, MN, "'Department of Cardiology, University Hospital Basel, Basel, Switzerland, and XUniversitäres Herz-Zentrum Bad Krozingen, Bad Krozingen, Germany. Funding: None.
} 
More than 7 million patients present annually to the emergency department (ED) with chest pain, ${ }^{1}$ and $>1$ million patients are hospitalized each year in the United States with acute myocardial infarction (AMI). ${ }^{2}$ The ability to rapidly exclude AMI through high-sensitivity troponin (hscTn) in combination with clinical evaluation may reduce ED length of stay, reduce financial cost, and improve outcomes in these challenging patients. Evidence suggests that even minimal elevations of conventional troponin (cTn) are associated with worse clinical outcome and that these patients may benefit from initiation of appropriate medical intervention. ${ }^{3,4}$ Furthermore, use of a very low cut-point for hs-cTn has been suggested as a tool to rule out AMI due to the resulting high negative predictive value (NPV). ${ }^{5}$ However, the introduction of hs-cTn may significantly decrease specificity and can prompt a costly cardiovascular workup in patients in which cTn is elevated due to nonischemic causes for cTn release. Although multiple studies have compared the diagnostic and prognostic test characteristics of cTn and hs-cTn, the results of these data are mixed. Therefore, we performed a diagnostic and prognostic collaborative meta-analysis to assess cTn values and hs-cTn values in patients with chest pain.

\section{Methods}

\section{Data sources and searches}

Two independent reviewers (M.J.L. and N.C.B.) systematically searched (November 2013) Cochrane CENTRAL, EMBASE, and MEDLINE/PubMed for studies that assessed both cTn and hs-cTn in patients with nontraumatic chest pain. Search criteria included "high sensitivity troponin" AND ("chest pain" OR "acute coronary syndromes" [ACS] OR "myocardial infarction"). We limited our search to studies published in peer-reviewed journals; trials presented in abstract-only form were excluded. Our meta-analysis was performed in accordance with the Meta-Analysis of Observational Studies in Epidemiology guidelines. ${ }^{6}$ After obtaining full reports, eligibility was assessed from the fulltext articles with divergences resolved after consensus. No extramural funding was used to support this work. The authors are solely responsible for the design and conduct of this study, all study analyses, the drafting and editing of the manuscript, and its final contents.

\section{Study selection}

Prespecified inclusion and exclusion criteria were established at study onset. We included any study that (a) assessed patients with nontraumatic chest pain and $(b)$ measured both cTn and hs-cTn levels. We excluded any study that (a) limited patients to only those with myocardial infarction (MI) or a specific subgroup of patients, (b) excluded patients with a baseline positive troponin, and (c) used a case-control format. We included studies regardless of whether patients with ST-segment elevation MI (STEMI) were included or excluded, whether the criterion standard diagnosis was made centrally or locally, and regardless of the cTn criteria used for diagnosis of AMI.

\section{Data extraction and quality assessment}

Data were abstracted by the same 2 investigators (M.J.L. and N.C.B.). An attempt was made to contact the corresponding authors of included studies to obtain complete data. Study quality was appraised in accordance with QUality Assessment of Diagnostic Accuracy Studies (QUADAS)-2. ${ }^{7}$ We accepted the authors' definitions of conventional and hs-cTn.

\section{Data synthesis and analysis}

Dichotomous variables are reported as proportions (percentages), whereas continuous variables are reported as mean (SD) or median. Sensitivity, specificity, positive predictive values (PPV), NPVs, positive and negative likelihood ratios (LRs), and diagnostic odds ratios (ORs) were computed. Pooling was performed using randomeffects methods. Measures of test performance are reported as point estimates (with 95\% CIs). These were calculated for the baseline cTn at presentation, baseline hs-cTn at presentation, cTn at the second serial sampling (second cTn), and hs-cTn at the second serial sampling (second hscTn). Adjudication of AMI was typically defined by cTn. Given that authors used their own cut-points and delta changes in troponin with different times for sampling, we were unable to assess for value of serial sampling in this meta-analysis.

We generated weighted symmetric summary receiver operating characteristic (SROC) plots using the MosesShapiro-Littenberg method. ${ }^{8}$ Area under the ROC curves of individual studies were pooled using a random effect generic-inverse variance method. Sources of clinical and statistical heterogeneity were explored by means of subgroup analyses and meta-regression with unrestricted maximum-likelihood meta-regression (inverse varianceweighted regression) on diagnostic ORs.

Binary outcomes from individual studies were combined with random-effect models, leading to computations of ORs with 95\% CIs. Between-study statistical heterogeneity was assessed using the Cochran $Q \chi^{2}$ test. $I^{2}$ was calculated as a measure of statistical heterogeneity; $I^{2}$ values of $25 \%, 50 \%$, and $75 \%$ represented mild, moderate, and severe inconsistency, respectively. Small study or publication bias was explored with funnel plots and Peters test. ${ }^{9}$ Statistical analysis was performed using Review Manager (RevMan) 5 version 5.1.7 freeware package (The Nordic Cochrane Centre, The Cochrane Collaboration, 2008, Copenhagen, Denmark), Meta-DiSc software, ${ }^{10}$ and NCSS 2007 (Kaysville, UT), with statistical significance for hypothesis testing set at the .05 two-tailed level and for heterogeneity testing at the .10 two-tailed level.

\section{Results}

Of the 824 citations we identified, we assessed 177 abstracts from which we performed detailed review of 91 


\section{Figure 1}

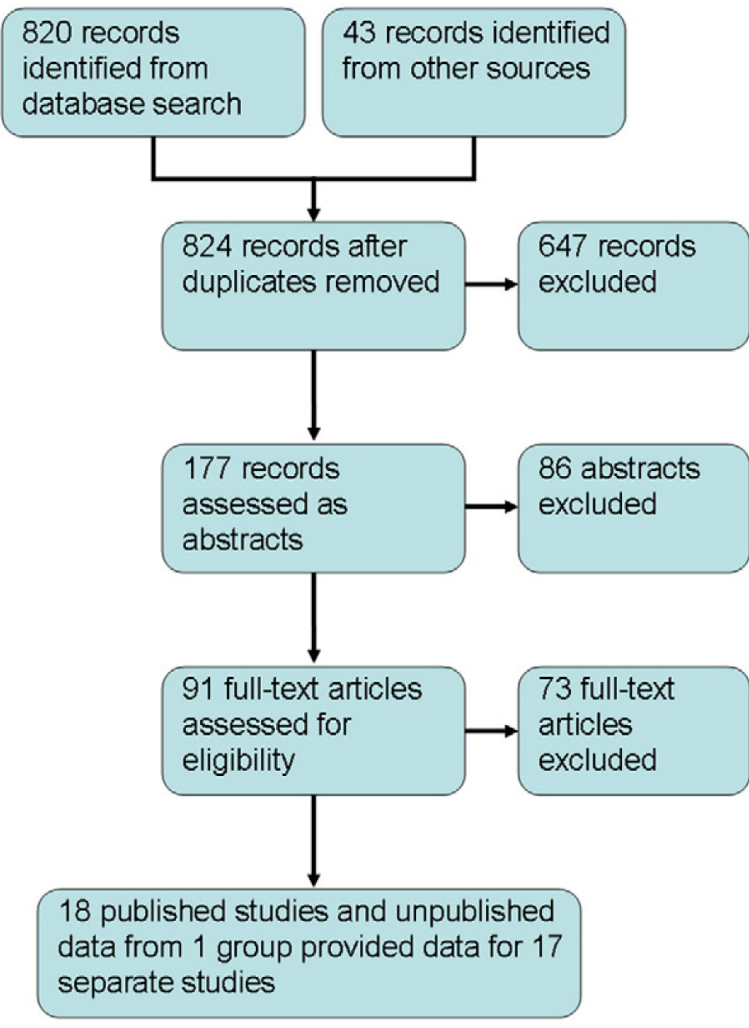

Flow diagram of study selection.

full-text manuscripts. Articles were excluded if the study was limited to only patients with stable coronary artery disease or only patients with ACS, patient duplication, exclusion of patients with baseline positive troponins, use of a casecontrol design, lack of or inadequate cTn data, and lack of adjudication data for AMI (excluded studies are listed in the supplement). Authors of the APACE study (Drs Twerenbold and Mueller) provided comprehensive data not only for the patients published in Haaf et al ${ }^{11}$ but also on an additional 416 patients to provide the most updated data from their registry. Thus, our systematic review and collaborative metaanalysis comprises data from 18 published studies ${ }^{12-29}$ (data from 3 studies were used to compile the findings of Aldous et al. ${ }^{12-14}$ ) and updated data from the APACE study to provide comprehensive data on 17 studies. The details of our flow diagram can be found in Figure 1. Study characteristics are presented in Table I, and appraisal of diagnostic study quality can be found $\mathrm{i}$

Supplementary Table I.

The 17 studies included a total of 8,644 patients (median of 332 patients [range 58-1,818]). Patient characteristics are shown in Table II. The population had a weighted mean age of $62 \pm 15$ years, $63 \%$ of patients were male, and there was a typical distribution of cardiovascular risk factors. Of the included patients, 20.7\% were diagnosed as having AMI, with $5.2 \%$ admitted with STEMI. In studies that reported unstable angina, $13.4 \%$ of patients were diagnosed as having unstable angina. Most studies used cTn levels for the adjudication of AMI, whereas several studies used a combination of cTn and hs-cTn levels

Supplementary Table I).

Diagnostic performance of individual studies is summarized for baseline cTn and baseline hs-cTn

Supplementary Table II), along with the second cTn and the second hs-cTn

Supplementary Table III). In addition to adjudicating AMI with conventional cTn, 6 studies also performed separate adjudication for AMI using the hs-cTn levels as the criterion standard to define AMI, and diagnostic performance for baseline cTn and hs-cTn is provided (online

Supplementary Table IV). Finally, the area under the ROC curves for baseline cTn, baseline hs-cTn, second cTn, and second hs-cTn for diagnosis of AMI can be found

Supplementary Table V.

\section{Diagnostic accuracy of cTn and hs-cTn}

The assays used in each study are shown in Table I. As seen in Table III, baseline hs-cTn had significantly greater sensitivity $(P<.001)$ and NPV $(P<.001)$, and significantly lower negative LR $(P<.01)$, whereas baseline $c$ Tn had significantly greater specificity $(P<.001)$, PPV $(P<.001)$, and positive LR $(P<.01)$. The SROC curves suggest a trend toward better diagnostic accuracy with baseline hsc'Tn (Table III, Figure 2). Comparison of pooled area under the ROC curves also suggested a trend toward better performance for baseline hs-cTn compared with baseline cTn $(0.91$ [95\% CI 0.89-0.93] vs 0.89 [95\% CI 0.86-0.91], respectively; $P=.22, I^{2}=33 \%$ ).

The second cTn was checked $2.6 \pm 1.5$ hours after the baseline cTn, and the second hs-cTn was checked $2.5 \pm 1.4$ hours after the baseline hs-cTn in 10 studies with 5,174 patients 1 Supplementary Table III). These data demonstrated that the sensitivity remained significantly greater for the second hs-cTn compared with the second cTn $(P<.05)$, whereas the second cTn had significantly greater specificity $(P<.001)$, PPV $(P<.001)$, and positive LR $(P<.01)$ compared with the second hs-cTn (Table III). Summary receiver operating characteristic curves demonstrated no difference in diagnostic accuracy (Table III). Pooled area under the ROC curve was not significantly different between the second cTn and the second hs-cTn (0.95 [95\% CI $0.93-0.97$ ] vs 0.96 [95\% CI $0.94-0.97]$, respectively; $P=.42, I^{2}=0 \%$ ) Supplementary Table V). Sensitivity analyses of conventional cTn or hs-cTn with exclusion of one study at a time did not appear to significantly change the sensitivity or specificity.

\section{Meta-regression analysis}

Meta-regression demonstrated that time from onset of chest pain to presentation was significantly associated with improved test performance for baseline cTn (regression coefficient $0.61 \pm \mathrm{SE} 0.20, P=.02$ ) but not 
Table I. Study characteristics

\begin{tabular}{|c|c|c|c|c|c|c|c|}
\hline Study & $\begin{array}{c}\text { Year } \\
\text { published }\end{array}$ & Patients & Centers & $\begin{array}{l}\text { Inclusion criteria } \\
\text { for chest pain }\end{array}$ & $\begin{array}{c}\text { Conventional Tn assay } \\
\text { (cut-point) }\end{array}$ & $\begin{array}{l}\text { HS-Tn assay } \\
\text { (cut-point) }\end{array}$ & $\begin{array}{l}\text { Follow- } \\
\text { Up (mo) }\end{array}$ \\
\hline Aldous et $a^{15}$ & 2012 & 939 & Multi & No exclusion & $\begin{array}{l}\text { Abbott Architect cTnl, } \\
30 \text { ng/L (10\% CV, } 32 \text { ng/L) }\end{array}$ & $\begin{array}{l}\text { Roche HS TnT, } \\
14 \mathrm{ng} / \mathrm{L} \text { (99th percentile) }\end{array}$ & 12 \\
\hline Aldous et al ${ }^{12-14}$ & 2011 & 332 & Single & No exclusion & $\begin{array}{l}\text { Abbott Architect cTnl, } \\
30 \mathrm{ng} / \mathrm{L}(10 \% \mathrm{CV}, 32 \mathrm{ng} / \mathrm{L})\end{array}$ & $\begin{array}{l}\text { Roche HS TnT, } \\
14 \mathrm{ng} / \mathrm{L} \text { (99th percentile) }\end{array}$ & 24 \\
\hline APACE & N/A & 1533 & Multi & $<12 \mathrm{~h}$ & $\begin{array}{l}\text { Roche cTnT } 4 \text { th gen, } \\
35 \mathrm{ng} / \mathrm{L}(10 \% \mathrm{CV}) \text { but } \\
\text { Siemens } \mathrm{RxL} \text { Tnl, } \\
140 \mathrm{ng} / \mathrm{L}(10 \% \mathrm{CV}) \text { to define AMI }\end{array}$ & $\begin{array}{l}\text { Roche HS TnT, } \\
14 \mathrm{ng} / \mathrm{L} \text { (99th percentile) }\end{array}$ & 24 \\
\hline Christ et al ${ }^{16}$ & 2010 & 137 & Single & No exclusion & $\begin{array}{l}\text { Roche cTnT 4th gen, } \\
35 \mathrm{ng} / \mathrm{L}(10 \% \mathrm{CV})\end{array}$ & $\begin{array}{l}\text { Roche HS TnT, } \\
14 \mathrm{ng} / \mathrm{L} \text { (99th percentile) }\end{array}$ & 8 \\
\hline Collinson et al ${ }^{17}$ & 2013 & 850 & Multi & No exclusion & $\begin{array}{l}\text { Siemens Stratus CS cTnl, } \\
70 \mathrm{ng} / \mathrm{L} \text { (99th percentile) }\end{array}$ & $\begin{array}{l}\text { Beckman AccuTnl, } \\
40 \mathrm{ng} / \mathrm{L} \text { (99th percentile) }\end{array}$ & 3 \\
\hline Eggers et $a^{18}$ & 2012 & 360 & Multi & $<8 h$ & $\begin{array}{l}\text { Siemens Stratus CS cTnl, } \\
70 \mathrm{ng} / \mathrm{L} \text { (99th percentile) }\end{array}$ & $\begin{array}{l}\text { Roche HS TnT, } \\
14 \mathrm{ng} / \mathrm{L} \text { (99th percentile) }\end{array}$ & 6 \\
\hline Freund et al ${ }^{19}$ & 2011 & 317 & Multi & $<6 \mathrm{~h}$ & $\begin{array}{l}\text { Siemens Xpand HM cTnl, } \\
140 \mathrm{ng} / \mathrm{L} \text { or Beckman Coulter } \\
\text { Access cTnl, } 60 \mathrm{ng} / \mathrm{L} \text { (both } 10 \% \mathrm{CV} \text { ) }\end{array}$ & $\begin{array}{l}\text { Roche HS TnT, } \\
14 \mathrm{ng} / \mathrm{L} \text { (99th percentile) }\end{array}$ & 1 \\
\hline $\begin{array}{l}\text { Hammerer- } \\
\text { Lercher et al }^{20}\end{array}$ & 2013 & 440 & Single & No exclusion & $\begin{array}{l}\text { Roche Elecsys cTnT } 4 \text { th gen, } \\
10 \mathrm{ng} / \mathrm{L} \text { (99th percentile) }\end{array}$ & $\begin{array}{l}\text { Roche HS TnT, } \\
14 \text { ng/L (99th percentile) }\end{array}$ & 8 \\
\hline Inoue et $\left.a\right|^{21}$ & 2011 & 283 & Multi & $<24 \mathrm{~h}$ & $\begin{array}{l}\text { Roche cTnT 4th gen, } 35 \mathrm{ng} / \mathrm{L} \\
(10 \% \mathrm{CV} \text { ) but } 100 \mathrm{ng} / \mathrm{L} \\
\text { (WHO criteria) to define AMI }\end{array}$ & $\begin{array}{l}\text { Roche HS TnT, } \\
14 \mathrm{ng} / \mathrm{L} \text { (99th percentile) }\end{array}$ & No \\
\hline Keller et al ${ }^{22}$ & 2009 & 1818 & Multi & No exclusion & $\begin{array}{l}\text { Roche Elecsys cTnT } 4 \text { th gen, } \\
30 \mathrm{ng} / \mathrm{L}(10 \% \mathrm{CV}) \text {, but Siemens } \\
\mathrm{RxL} \text { Tnl, } 140 \mathrm{ng} / \mathrm{L}(10 \% \mathrm{CV}) \\
\text { to define AMl }\end{array}$ & $\begin{array}{l}\text { Siemens sensitive Tnl Ultra, } \\
40 \mathrm{ng} / \mathrm{L} \text { (99th percentile) }\end{array}$ & $\begin{array}{l}1 ; \text { unable } \\
\text { to abstract }\end{array}$ \\
\hline Lotze ef $\mathrm{al}^{23}$ & 2011 & 142 & Single & No exclusion & $\begin{array}{l}\text { Roche cTnT 4th gen, } \\
100 \mathrm{ng} / \mathrm{L} \text { (WHO Criteria) }\end{array}$ & $\begin{array}{l}\text { Roche HS TnT, } \\
14 \text { ng/L (99th percentile) }\end{array}$ & No \\
\hline Melki et al ${ }^{24}$ & 2011 & 233 & Single & $<12 \mathrm{~h}$ & $\begin{array}{l}\text { Roche cTnT } 4 \text { th gen, } \\
40 \mathrm{ng} / \mathrm{L}(10 \% \mathrm{CV}, 35 \mathrm{ng} / \mathrm{L})\end{array}$ & $\begin{array}{l}\text { Roche HS TnT, } \\
14 \text { ng/L (99th percentile) }\end{array}$ & No \\
\hline Meune et $a^{25}$ & 2011 & 58 & Single & $<6 \mathrm{~h}$ & $\begin{array}{l}\text { Siemens Xpand HM cTnl, } \\
70 \mathrm{ng} / \mathrm{L} \text { (99th percentile) }\end{array}$ & $\begin{array}{l}\text { Roche HS TnT, } \\
14 \mathrm{ng} / \mathrm{L} \text { (99th percentile) }\end{array}$ & No \\
\hline Pracon et $a^{26}$ & 2012 & 187 & Single & $<24 \mathrm{~h}$ & $\begin{array}{l}\text { Siemens Dimension Flex Tnl, } \\
70 \mathrm{mg} / \mathrm{L} \text { (99th percentile) }\end{array}$ & $\begin{array}{l}\text { Abbott Architect Stat Tnl, } \\
28 \mathrm{ng} / \mathrm{L} \text { (99th percentile) }\end{array}$ & No \\
\hline Santalo et $a^{27}$ & 2013 & 356 & Multi & No exclusion & $\begin{array}{l}\text { Roche Cobas e401 cTnT } 4 \text { th gen, } \\
10 \mathrm{ng} / \mathrm{L} \text { (99th percentile) }\end{array}$ & $\begin{array}{l}\text { Roche HS TnT, } \\
14 \mathrm{ng} / \mathrm{L} \text { (99th percentile) }\end{array}$ & 12 \\
\hline Schreiber et al ${ }^{28}$ & 2012 & 465 & Single & No exclusion & $\begin{array}{l}\text { Siemens Dimension RxL Tnl, } \\
140 \text { ng/L (10\% CV) }\end{array}$ & $\begin{array}{l}\text { Singulex Erenna HS-Tnl, } \\
8 \mathrm{ng} / \mathrm{L}(99 \mathrm{th} \text { percentile, } \\
10.1 \mathrm{ng} / \mathrm{L})\end{array}$ & 1 \\
\hline Sebbane et $a^{29}$ & 2013 & 194 & Single & $<12 \mathrm{~h}$ & $\begin{array}{l}\text { Beckman Access } 2 \text { cTnl, } \\
40 \text { ng/L (intended 99th percentile) }\end{array}$ & $\begin{array}{l}\text { Roche HS TnT, } \\
14 \mathrm{ng} / \mathrm{L} \text { (99th percentile) }\end{array}$ & No \\
\hline
\end{tabular}

Abbott (Abbott Park, IL), Roche (Indianapolis, IN), Siemens (Tarrytown, NY), Singulex (St Louis, MO).

baseline hs-cTn (regression coefficient $0.38 \pm$ SE $0.20, P=$ .10). Neither time from presentation to the second cTn nor the second hs-cTn was significantly associated with test performance. The percentage of patients with STEMI (regression coefficient $-4.6 \pm 1.1, P=.001$ ), male sex (regression coefficient $-8.3 \pm$ SE 3.0, $P=.02$ ), diabetes (regression coefficient $-8.0 \pm \mathrm{SE} 2.9, P=.02$ ), and prevalence of AMI (regression coefficient $-3.2 \pm \mathrm{SE} 1.2, P$ $=.02$ ) were significantly associated with test performance for baseline cTn but was not associated with test performance for baseline hs-cTn. Age, creatinine levels, and estimated glomerular filtration rate were not associated with test performance for baseline cTn or baseline hs-cTn. The definition of the delta, or the change by rise and/or fall of troponin, used to diagnosis AMI was also not significantly associated with test performance.

\section{Subgroup analysis}

When comparing studies that used the $10 \%$ coefficient variance (CV) cut-point ${ }^{12,15,16,19,22,24,28}$ (see also APACE) vs 99th percentile cut-point ${ }^{17,18,20,25-27,29}$ for cTn to define AMI, baseline cTn using 10\% CV cut-point had significantly greater specificity $(0.957[0.950-0.962]$ vs $0.921[0.908-0.933])$, PPV (0.813 [0.788-0.836] vs 0.699 [0.657-0.738]), and positive LR (15.804 [10.699-23.345] vs $8.905[5.771-13.740]$ ) than baseline cTn using 99th percentile cut-point, with no significant differences between the groups in terms of sensitivity $(0.754$ [0.728-0.778] vs 0.788 [0.747-0.824]), NPV (0.940 [0.932-0.946] vs 0.949 [0.938-0.959]), negative LR (0.260 [0.218-0.311] vs 0.238 [0.192-0.294]), diagnostic OR (60.651 [36.377-101.12] vs 44.054 [26.685-72.727]), or SROC $(0.889[0.756-0.990]$ vs 0.919 [0.879-0.959]). 
Table II. Patient characteristics of included studies

\begin{tabular}{|c|c|c|c|c|c|c|c|c|c|c|c|c|c|}
\hline Study & Age (y) & Male & Prior CAD & Prior MI & HTN & HLD & DM & Smoking & TTP (h) & STEMI & NSTEMI & AMI & UA \\
\hline Aldous et al $2012^{15}$ & 65 & $59.7 \%$ & $\%$ & NR & $60.8 \%$ & $7.6 \%$ & $16.5 \%$ & $60.6 \%$ & 6.3 & 0 & $21.8 \%$ & $21.8 \%$ & NR \\
\hline Aldous et al $2011^{12-14}$ & 64 & $60.2 \%$ & $53.9 \%$ & NR & $45.8 \%$ & $38.0 \%$ & $16.3 \%$ & $17.2 \%$ & 4 & 0 & $33.1 \%$ & $33.1 \%$ & $17.2 \%$ \\
\hline APACE & $63 \pm 16$ & $67.0 \%$ & $36.2 \%$ & $24.2 \%$ & $65.9 \%$ & $50.8 \%$ & $19.2 \%$ & $24.1 \%$ & 5 & $3.7 \%$ & $11.5 \%$ & $15.3 \%$ & $14.3 \%$ \\
\hline Christ et al ${ }^{16}$ & $66 \pm 16$ & $63.5 \%$ & $34.3 \%$ & $32.8 \%$ & $66.4 \%$ & $35.0 \%$ & $22.6 \%$ & $21.9 \%$ & NR & $2.9 \%$ & $11.7 \%$ & $14.6 \%$ & $19.0 \%$ \\
\hline Collinson ef al ${ }^{17}$ & 54 & $59.6 \%$ & NR & $5.8 \%$ & $35.4 \%$ & $23.6 \%$ & $8.1 \%$ & $28.5 \%$ & 5.9 & 0 & $8.0 \%$ & $8.0 \%$ & NR \\
\hline Eggers et $a^{18}$ & $66 \pm 12$ & $65.6 \%$ & $42.8 \%$ & $37.5 \%$ & $42.8 \%$ & $38.3 \%$ & $18.3 \%$ & $18.1 \%$ & 4.5 & 0 & $35.6 \%$ & $35.6 \%$ & $18.9 \%$ \\
\hline Freund et al ${ }^{19}$ & $57 \pm 17$ & $64.7 \%$ & $31.6 \%$ & $26.2 \%$ & $36.6 \%$ & $35.8 \%$ & $13.9 \%$ & $40.6 \%$ & NR & $4.1 \%$ & $10.1 \%$ & $14.2 \%$ & $3.5 \%$ \\
\hline $\begin{array}{l}\text { Hammerer- } \\
\text { Lercher et al }^{20}\end{array}$ & $56 \pm 20$ & $52.3 \%$ & $19.1 \%$ & NR & $46.4 \%$ & NR & $7.5 \%$ & NR & 3 & $\%$ & $32 \%$ & $9.1 \%$ & NR \\
\hline Inove et al & 12 & $\%$ & $\Lambda$ & $\Lambda$ & 5 & $2 \%$ & 2 & & 3 & & & $6 \%$ & $10.2 \%$ \\
\hline Keller et al ${ }^{22}$ & $61 \pm 14$ & $66.4 \%$ & $35.8 \%$ & NR & $73.7 \%$ & $73.0 \%$ & $15.7 \%$ & $24.3 \%$ & NR & $7.2 \%$ & $15.6 \%$ & $22.7 \%$ & $13.2 \%$ \\
\hline Lotze ef $a^{23}$ & $71 \pm 14$ & $76.0 \%$ & $27.5 \%$ & $15.5 \%$ & $73.9 \%$ & $16.9 \%$ & $28.9 \%$ & $7.7 \%$ & NR & $6.3 \%$ & $2.8 \%$ & $9.2 \%$ & $2.1 \%$ \\
\hline Melki et al ${ }^{24}$ & 65 & $66.5 \%$ & NR & $30.0 \%$ & $50.2 \%$ & NR & $22.7 \%$ & $17.2 \%$ & 5.3 & 0 & $48.9 \%$ & $48.9 \%$ & $12.0 \%$ \\
\hline Meune et $a^{25}$ & $58 \pm 14$ & $63.8 \%$ & NR & $20.7 \%$ & $46.7 \%$ & $37.9 \%$ & $22.4 \%$ & $32.8 \%$ & 7.5 & 0 & $22.4 \%$ & $22.4 \%$ & $29.3 \%$ \\
\hline Pracon et $a^{26}$ & $64 \pm 14$ & $63.6 \%$ & NR & $17.6 \%$ & $61.0 \%$ & $36.4 \%$ & $14.4 \%$ & $13.9 \%$ & NR & $23.0 \%$ & $21.9 \%$ & $44.9 \%$ & $5.9 \%$ \\
\hline Santalo et $\mathrm{al}^{27}$ & 69 & $67.9 \%$ & $34.9 \%$ & NR & $62.0 \%$ & NR & $26.4 \%$ & NR & 5 & 0 & $21.9 \%$ & $21.9 \%$ & $29.5 \%$ \\
\hline Schreiber et al ${ }^{28}$ & 67 & $49.2 \%$ & NR & $19.1 \%$ & $62.2 \%$ & NR & $17.4 \%$ & $11.2 \%$ & NR & 0 & $2.6 \%$ & $2.6 \%$ & $3.4 \%$ \\
\hline Sebbane et $\mathrm{al}^{29}$ & $61=$ & $63.4 \%$ & $21.6 \%$ & & $34.0 \%$ & & $14.1 \%$ & $36.6 \%$ & 4.24 & & $12.4 \%$ & $26.3 \%$ & $16.0 \%$ \\
\hline Weighted mean & $62 \pm 15$ & $63.4 \%$ & $37.5 \%$ & $20.9 \%$ & $58.1 \%$ & $50.1 \%$ & $16.8 \%$ & $28.3 \%$ & $5.1 \pm 1.1$ & $5.2 \%$ & $15.5 \%$ & $20.7 \%$ & $13.4 \%$ \\
\hline
\end{tabular}

Abbreviations: CAD, Coronary artery disease; HTN, hypertension; HLD, hyperlipidemia; DM, diabetes mellitus; TTP, time from onset of chest pain to presentation; NSTEMI, non-ST elevation $\mathrm{Ml}$; UA, unstable angina.

Table III. Summary of sensitivity, specificity, PPV, NPV, positive LR, negative LR, diagnostic OR (DOR), and area under the SROC curves for the baseline and second serial conventional and hs-cTn (hs-cTn) for AMI

\section{Baseline cTn}

$0.749(0.728-0.769)$

$0.938(0.932-0.943)$

$0.759(0.738-0.778)$

$0.935(0.929-0.940)$

$9.913(6.648-14.781)$

$0.262(0.217-0.317)$

$41.665(24.732-70.191)$

$0.890(0.839-0.941)$
Second Serial cTn

Second Serial hs-cTn

Pooled sensitivity

Pooled PPV

Pooled NPV

Summary positive $L R$

Summary DOR

Area under the SROC curve
Baseline hs-cTn

$0.884(0.868-0.898)$

$0.816(0.807-0.826)$

$0.558(0.539-0.576)$

$0.964(0.959-0.969)$

$4.393(3.403-5.673)$

$0.156(0.116-0.210)$

$32.609(20.477-51.931)$

$0.923(0.899-0.947)$
0.895 (0.867-0.919)

$0.952(0.944-0.959)$

$0.758(0.724-0.790)$

$0.982(0.977-0.986)$

$13.163(7.667-22.596)$

$0.137(0.092-0.204)$

$95.503(45.727-199.46)$

$0.951(0.919-0.983)$
$0.928(0.903-0.948)$

$0.807(0.794-0.821)$

$0.443(0.414-0.472)$

$0.985(0.980-0.990)$

$4.663(3.576-6.080)$

$0.112(0.069-0.182)$

$49.716(25.238-97.938)$

$0.948(0.912-0.984)$
There was no significant difference in test performance for baseline cTn in studies that used a $10 \% \mathrm{CV}$ cut-point compared with a 99th percentile cut-point to define AMI as assessed by pooled area under the ROC curves $(0.90$ [0.86-0.93] vs 0.91 [0.88-0.93], $P=.61, I^{2}=0 \%$ ).

When comparing the diagnostic performance of baseline $\mathrm{cTnT}^{16,20,23,24,27}$ (see also APACE) and cTnI $^{12,15,17-19,25,26,28,29}$ to define AMI, baseline cTnT had significantly lower specificity (0.931 [0.920-0.941] vs $0.950[0.941-0.957])$ and PPV (0.701 [0.661-0.740] vs 0.790 [0.759-0.820]) compared with baseline cTnI. There were no differences between baseline cTnT and baseline cTnI in sensitivity $(0.758[0.717-0.795]$ vs 0.790 [0.759-0.820]), NPV (0.947 [0.938-0.956] vs 0.950 [0.9410.957]), positive LR (8.822 [3.996-19.478] vs 12.532 [7.848-20.010]), negative LR (0.263 [0.20-0.314] vs 0.235 [0.189-0.292]), diagnostic OR (42.289 [21.696-82.428] vs 57.519 [32.471-101.89]), or SROC (0.904 [0.860-0.948] vs $0.917[0.863-0.971])$. There was no significant difference in test performance for baseline cTnT and baseline cTnI as assessed by pooled area under the ROC curves $(0.89$ [0.86-0.93] vs $\left.0.91[0.89-0.93], P=.30, I^{2}=7.1 \%\right)$.

\section{AMI definition based on hs-cTn}

When limiting studies to those that provided a separate adjudication using hs-cTn to define AMI, ${ }^{12,14,16,17,24,27}$ (see also APACE), the mean prevalence of AMI increased from $23 \% \pm 15 \%$ when AMI was defined by cTn to $29.6 \% \pm 16.5 \%$ when AMI was defined by hs-cTn. When AMI was defined by hs-cTn, the baseline hs-cTn had significantly greater test performance based on pooled area under the ROC curves compared with baseline cTn $(0.91$ [95\% CI 0.88-0.94] vs 0.80 [95\% CI 0.74-0.87], respectively; $P=.004)$. Baseline cTn had a significant reduction in sensitivity $(0.666$ vs $0.749, P<$ $.001)$ and NPV (0.906 vs $0.935, P<.001)$ when AMI was defined by hs-cTn compared with when AMI was defined by cTn. Baseline hs-cTn also had a significant reduction in sensitivity $(0.857$ vs $0.884, P<.05)$ and NPV $(0.953$ vs 0.964 

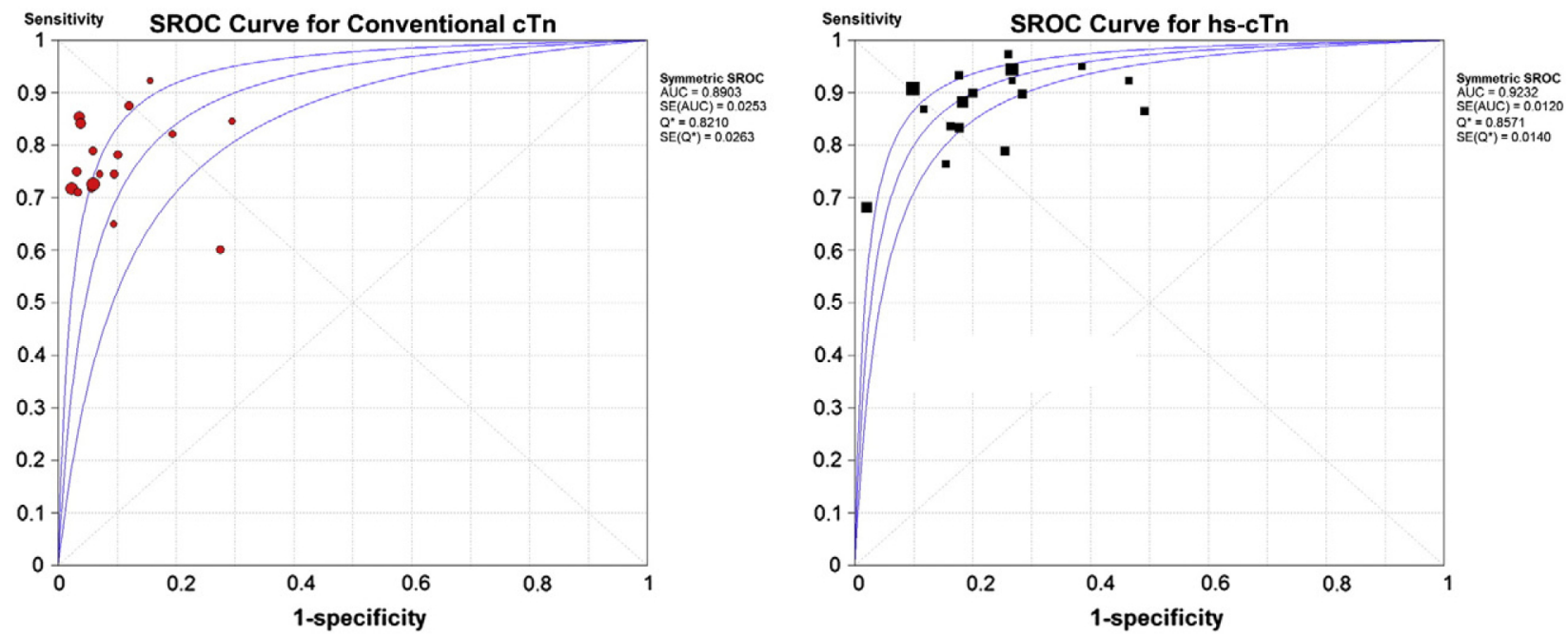

Summary ROC curves for the baseline conventional cTn (left) and baseline hs-cTn (right), which plots sensitivity and 1 - specificity for each study, enabling comparison of the 2 assays. Studies were weighted by least-squares method using the inverse variance. Studies are plotted for conventional cTn with red circles and plotted for hs-cTn with black squares. Symmetric SROC curves are present with a 95\% Cl, and area under the SROC curve is provided along with SEs to the right in each figure.

Table IV. Summary of sensitivity, specificity, PPV, NPV, positive $L R$, negative $L R$, diagnostic $O R(D O R)$, and area under the summary SROC curves for cTn and hs-cTn when AMl is based on using the cut-point for hs-cTn

Baseline cTn

$0.666(0.631-0.699)$

$0.941(0.931-0.950)$

$0.768(0.734-0.799)$

$0.906(0.894-0.916)$

$8.797(3.892-19.888)$

$0.314(0.205-0.479)$

$0.145(0.070-0.304)$

$30.004(14.080-63.937) \quad 57.034(24.958-130.33)$

$0.904(0.817-0.991)$

\section{Baseline hs-cTn}

$0.857(0.830-0.881)$

$0.854(0.840-0.868)$

$0.632(0.602-0.661)$

$0.953(0.944-0.962)$

$7.482(4.114-13.608)$

positive LR

negative $L R$

Summary DOR

Area under the SROC curve

$0.945(0.907-0.983)$
$P<.05)$ with an increase in specificity ( 0.854 vs $0.816, P<$ $.001)$ and PPV (0.632 vs $0.558, P<.001)$ when AMI was defined by hs-cTn compared with when AMI was defined by cTn (Table IV

When strictly applying the definition of hs-cTn measuring the 99th percentile upper reference limit with an analytical imprecision of $<10 \%,{ }^{30,31}$ Keller et $\mathrm{al}^{22}$ and Pracon et $\mathrm{al}^{26}$ are no longer considered under the category of hs-cTn. Therefore, we repeated the previous analysis with 15 studies to determine whether this significantly affected our previous findings. When using studies that used strict hs-cTn assays, baseline cTn and hs-cTn had similar values to those before in regard to sensitivity $(0.752[0.727-0.775]$ vs $0.877[0.857-$
$0.894])$, specificity $(0.939[0.933-0.946]$ vs $0.793[0.782$ 0.803]), PPV (0.750 [0.725-0.773] vs 0.505 [0.484-0.526]), NPV (0.940 [0.933-0.946] vs 0.964 [0.958-0.969]), positive LR (10.366 [6.475-16.595] vs 4.002[3.203-4.999]), negative LR (0.259 [0.204-0.329] vs 0.164 [0.119-0.225]), diagnostic OR (44.019 [23.073-83.983] vs 28.645 [18.135-45.247]), and SROC (0.893 [0.835-0.951] vs 0.916 [0.888-0.944]). Using a strict definition of hs-cTn compared with the study-defined hs-cTn (Table III) lowered specificity $(0.793$ vs 0.816 , respectively; $P<.01)$ and PPV ( 0.505 vs 0.558 , respectively; $P<.01)$ but was not significantly associated with sensitivity, NPV, positive LR, negative LR, diagnostic OR, or area under the SROC curve.

\section{cTn and hs-cTn for prognosis}

Outcome data were provided for 10 studies only because data could not be accurately extracted from Keller et al. ${ }^{22}$ During a mean follow-up of 12.3 months (Table I), our study demonstrated that patients with an elevated baseline cTn or elevated baseline hs-cTn have significantly higher incidence of death nonfatal MI Supplementary Figure 1A), Supplementary Figure 1B), or their combination Supplementary Figure 1C) compared with patients who had a negative baseline cTn or negative baseline hs-cTn, respectively. The ORs for baseline cTn and baseline hs-cTn are not significantly different for the outcomes of death

Supplementary Figure $1 \mathrm{~A} ; P=.46, I^{2}=0 \%$ ), nonfatal MI Supplementary Figure $1 \mathrm{~B} ; P=.62, I^{2}=$ $0 \%$ ), or their combination Supplementary Figure $1 \mathrm{C} ; P=.75, I^{2}=0 \%$ ) during follow-up. However, 


\section{Figure 3}

A

\begin{tabular}{|c|c|c|c|c|c|}
\hline Study or Subgroup & Events & Total & Events & Total & Weight \\
\hline Aldous 2011 & 13 & 88 & 1 & 189 & $7.3 \%$ \\
\hline Aldous 2012 & 19 & 185 & 11 & 608 & $20.0 \%$ \\
\hline APACE & 44 & 197 & 23 & 966 & $23.4 \%$ \\
\hline Christ 2010 & 3 & 32 & 0 & 66 & $4.0 \%$ \\
\hline Collinson 2013 & 2 & 47 & 3 & 749 & $8.7 \%$ \\
\hline Eggers 2012 & 6 & 105 & 3 & 200 & $12.0 \%$ \\
\hline Freund 2011 & 2 & 23 & 0 & 160 & $3.8 \%$ \\
\hline Hammerer-Lercher 2013 & 4 & 83 & 12 & 324 & $14.7 \%$ \\
\hline Santalo 2013 & 3 & 89 & 1 & 205 & $6.2 \%$ \\
\hline Schreiber 2012 & 0 & 20 & 0 & 350 & \\
\hline Total $(95 \% \mathrm{Cl})$ & & 869 & & 3817 & $100.0 \%$ \\
\hline Total events & 96 & & 54 & & \\
\hline \multicolumn{6}{|c|}{$\begin{array}{l}\text { Heterogeneity: } \text { Tau }^{2}=0.39: \mathrm{Chi}^{2}=15.83, \mathrm{df}=8(P=.04): \mathrm{I}^{2}=49^{\circ} \\
\text { Test for overall effect: } Z=6.02(P<.00001)\end{array}$} \\
\hline
\end{tabular}

Odds Ratio

M-H, Random, $95 \% \mathrm{Cl}$

32.59 [4.19, 253.51]

$6.21[2.90,13.31]$

$11.79[6.92,20.08]$

$15.78[0.79,315.28]$

$11.05[1.80,67.82]$

$3.98[0.97,16.25]$

$37.33[1.73,803.70]$

$1.32[0.41,4.19]$

$7.12[0.73,69.38]$

Not estimable

$7.24[3.80,13.80]$
Odds Ratio

M.H, Random, $95 \% \mathrm{Cl}$

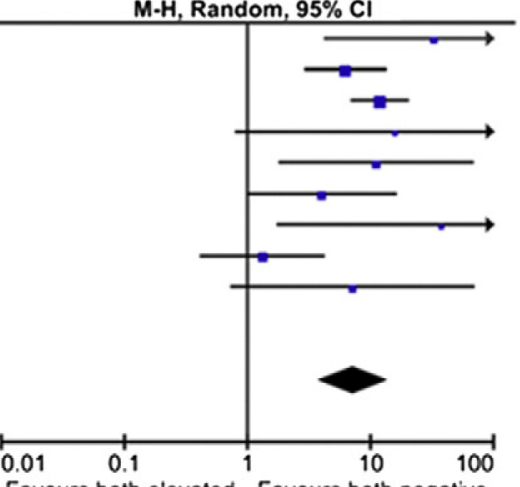

Favours both elevated Favours both negative

B

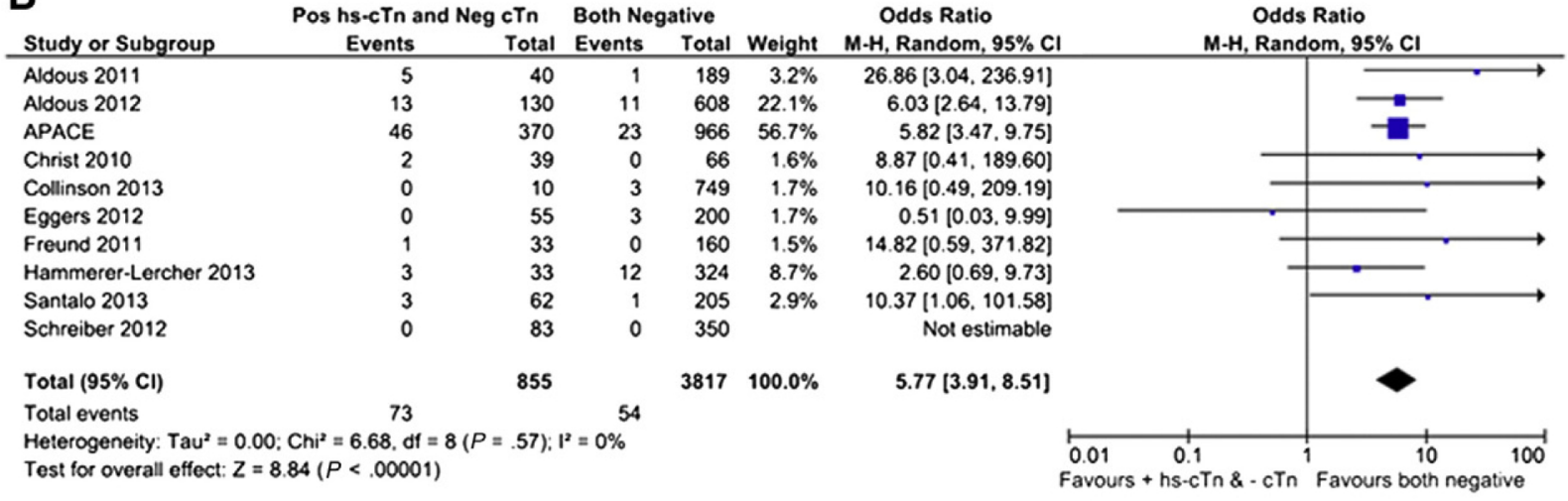

C

\begin{tabular}{|c|c|c|c|c|c|}
\hline \multirow[b]{2}{*}{ Study or Subgroup } & \multicolumn{2}{|c|}{ Both Elevated } & \multicolumn{2}{|c|}{ Pos hs-eTn and Neg cTn } & \multirow[b]{2}{*}{ Weight } \\
\hline & Events & Total & Events & Total & \\
\hline Aldous 2011 & 13 & 88 & 5 & 40 & $9.4 \%$ \\
\hline Aldous 2012 & 19 & 185 & 13 & 130 & $20.7 \%$ \\
\hline APACE & 44 & 197 & 46 & 370 & $55.2 \%$ \\
\hline Christ 2010 & 3 & 32 & 2 & 39 & $3.3 \%$ \\
\hline Collinson 2013 & 2 & 47 & 0 & 10 & $1.2 \%$ \\
\hline Eggers 2012 & 6 & 105 & 0 & 55 & $1.4 \%$ \\
\hline Freund 2011 & 2 & 23 & 1 & 33 & $1.9 \%$ \\
\hline Hammerer-Lercher 2013 & 4 & 83 & 3 & 33 & $4.7 \%$ \\
\hline Santalo 2013 & 3 & 89 & 1 & 62 & $2.2 \%$ \\
\hline Schreiber 2012 & 0 & 20 & 0 & 83 & \\
\hline Total $(95 \% \mathrm{CI})$ & & 869 & & 855 & $100.0 \%$ \\
\hline Total events & 96 & & 71 & & \\
\hline \multicolumn{6}{|c|}{ Heterogeneity: $\operatorname{Tau}^{2}=0.00 ; \mathrm{Chi}^{2}=6.17, \mathrm{df}=8(P=.63) ; \mathrm{I}^{2}=0 \%$} \\
\hline
\end{tabular}

Odds Ratio

Odds Ratio

M.H, Random, 95\% Cl

$1.21[0.40,3.67]$

$1.03[0.49,2.17]$

$2.03[1.28,3.20]$

$1.91[0.30,12.22]$

$1.15[0.05,25.86]$

$7.25[0.40,131.14]$

$3.05[0.26,35.77]$

$0.51[0.11,2.40]$

$2.13[0.22,20.95]$

Not estimable

$1.60[1.14,2.24]$

M-H, Random, $95 \% \mathrm{Cl}$

Forest plots comparing death during follow-up between patients with elevation of both baseline cTn and baseline hs-cTn and patients with both negative baseline cTn and baseline hs-cTn (A), death during follow-up between patients with elevation of baseline hs-cTn and negative baseline cTn and patients with both negative baseline cTn and baseline hs-cTn (B), and death during follow-up between patients with elevation of both baseline cTn and baseline hs-cTn and patients with elevation of baseline hs-cTn and negative baseline $\mathrm{cTn}(\mathrm{C})$ for patients that presented with chest pain.

significantly more individuals with an elevated baseline hscTn died ( 173 with elevated baseline hs-cTn died vs 105 with elevated baseline cTn died of the 231 total individuals who died during follow-up, $P<.001$ ) or developed AMI (143 with elevated baseline hs-cTn developed MI vs 92 with elevated baseline cTn developed MI of 222 total individuals who had AMI, $P<.001$ ) during follow-up compared with individuals with an elevated baseline cTn. 
A

\begin{tabular}{|c|c|c|c|c|c|c|c|c|}
\hline Study or Subgroup & \multicolumn{2}{|c|}{ Both Elevated } & \multicolumn{2}{|c|}{ Both Negative } & Weight & $\begin{array}{c}\text { Odds Ratio } \\
\text { M-H, Random, } 95 \% \mathrm{Cl}\end{array}$ & \multicolumn{2}{|c|}{$\begin{array}{c}\text { Odds Ratio } \\
\text { M-H, Random, } 95 \% \mathrm{Cl}\end{array}$} \\
\hline Aldous 2011 & 21 & 88 & 21 & 189 & $25.9 \%$ & $2.51[1.29,4.89]$ & & $\because-$ \\
\hline Aldous 2012 & 21 & 185 & 15 & 608 & $24.8 \%$ & $5.06[2.55,10.04]$ & & \\
\hline APACE & 19 & 197 & 30 & 966 & $31.3 \%$ & $3.33[1.83,6.05]$ & & $\rightarrow-$ \\
\hline Christ 2010 & 3 & 32 & $\mathbf{0}$ & 66 & $1.5 \%$ & $15.78[0.79,315.28]$ & & \\
\hline Collinson 2013 & 2 & 47 & 2 & 749 & $3.3 \%$ & $16.60[2.29,120.58]$ & & \\
\hline Eggers 2012 & 10 & 105 & 5 & 200 & $10.4 \%$ & $4.11[1.36,12.35]$ & & \\
\hline Santalo 2013 & 5 & 89 & 1 & 205 & $2.8 \%$ & $12.14[1.40,105.50]$ & & \\
\hline Total $(95 \% \mathrm{Cl})$ & & 743 & & 2983 & $100.0 \%$ & $3.93[2.73,5.66]$ & & \\
\hline Total events & 81 & & 74 & & & & & \\
\hline $\begin{array}{l}\text { Heterogeneity: } \mathrm{Tau}^{2} \\
\text { Test for overall effect }\end{array}$ & $\begin{array}{l}.02: \mathrm{Chi}^{2} \\
=7.33(P\end{array}$ & $\begin{array}{l}46 . d \\
.000\end{array}$ & $\begin{array}{l}f=6(P= \\
\text { 01) }\end{array}$ & 37): $1^{2}=$ & & & $\begin{array}{ll}0.01 & 0.1 \\
\text { Favours both elevated }\end{array}$ & $\begin{array}{cc}10 & 100 \\
\text { Favours both negative }\end{array}$ \\
\hline
\end{tabular}

B

\begin{tabular}{|c|c|c|c|c|}
\hline \multirow{2}{*}{ Study or Subgroup } & \multicolumn{2}{|c|}{ Pos hs-cTn and Neg cTn } & \multicolumn{2}{|c|}{ Both Negativ } \\
\hline & Events & Total & Events & $=$ \\
\hline Aldous 2011 & 10 & 40 & 21 & \\
\hline Aldous 2012 & 14 & 130 & 15 & \\
\hline APACE & 30 & 370 & 30 & \\
\hline Christ 2010 & 3 & 39 & 0 & \\
\hline Collinson 2013 & 0 & 10 & 2 & \\
\hline Eggers 2012 & 2 & 55 & 5 & \\
\hline Santalo 2013 & 1 & 62 & 1 & \\
\hline Total $(95 \% \mathrm{Cl})$ & & 706 & & \\
\hline Total events & 60 & & 74 & \\
\hline $\begin{array}{l}\text { Heterogeneity: Tau } \\
\text { Test for overall effec }\end{array}$ & $\begin{array}{l}00: \mathrm{Chi}^{2}=4.1 \\
=6.21(P<.\end{array}$ & $P=$ & $1^{2}=09$ & \\
\hline
\end{tabular}

Odds Ratio -H, Random, 95\% CI

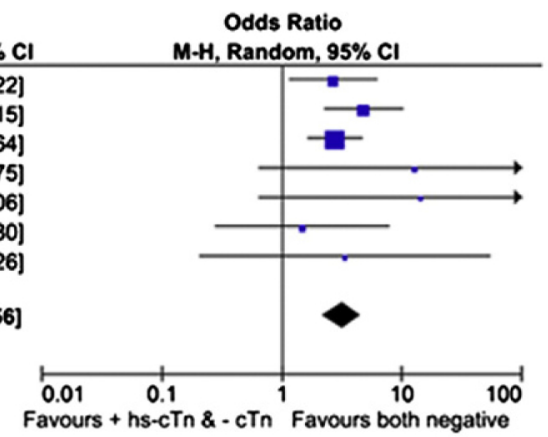

$2.67[1.14,6.22]$

$4.77[2.24,10.15]$

$2.75[1.63,4.64]$

$12.75[0.64,253.75]$

$14.24[0.64,315.06]$

$1.47[0.28,7.80]$

$3.34[0.21,54.26]$

$3.17[2.20,4.56]$

C

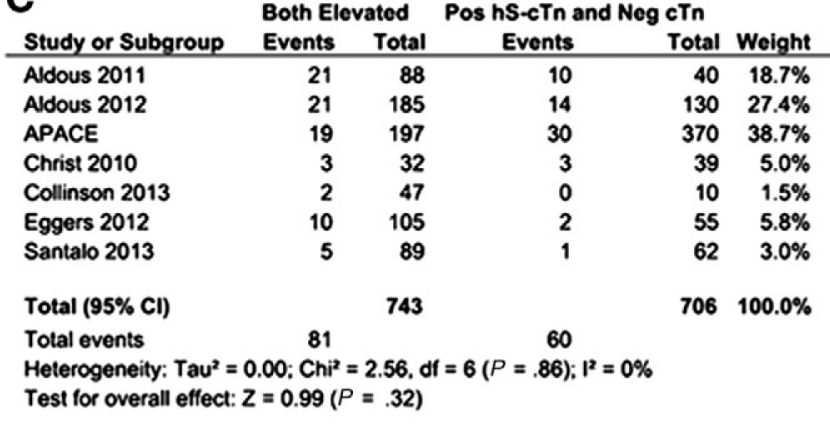

Odds Ratio Odds Ratio

M.H, Random, 95\% Cl

$0.94[0.39,2.24]$

$1.06[0.52,2.17]$

1.21 [0.66. 2.21]

$1.24[0.23 .6 .62]$

$1.15[0.05,25.86]$

$2.79[0.59,13.21]$

$3.63[0.41,31.87]$

$1.21[0.83,1.76]$

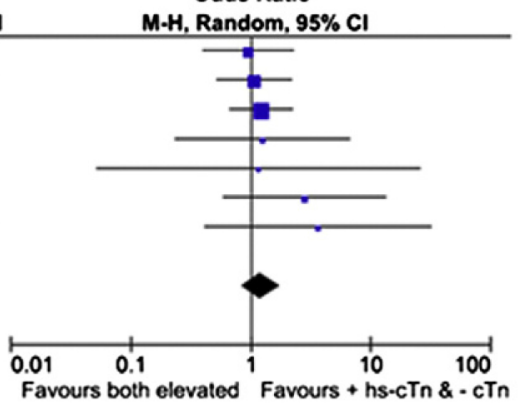

Forest plots comparing nonfatal MI during follow-up between patients with elevation of both baseline cTn and baseline hs-cTn and patients with both negative baseline cTn and baseline hs-cTn (A), nonfatal MI during follow-up between patients with elevation of baseline hs-cTn and negative baseline cTn and patients with both negative baseline cTn and baseline hs-cTn (B), and nonfatal Ml during follow-up between patients with elevation of both baseline cTn and baseline hs-cTn and patients with elevation of baseline hs-cTn and negative baseline cTn (C) for patients that presented with chest pain.

Patients who had elevation of both baseline cTn and baseline hs-cTn had significantly greater death (Figure 3A), nonfatal MI (Figure 4A), and their combination (Figure 5A) during follow-up compared with patients with both negative baseline cTn and baseline hs-cTn. Patients who had elevation of baseline hs-cTn but a negative baseline cTn had significantly greater death (Figure 3B), nonfatal MI (Figure 4B), and their combination (Figure 5B) during follow-up compared with patients with both negative baseline cTn and baseline hs-cTn. Patients with elevation of both baseline cTn and baseline hs-cTn had significantly greater death (Figure 3C) and the combination end point of death and nonfatal MI (Figure 5C) but no significant difference in nonfatal MI (Figure 4C) during follow-up compared with patients with an elevated baseline hs-cTn but a negative baseline cTn. Visual inspection of funnel plots along with Peters test did not show evidence of publication bias for baseline cTn (Peters test, $P=.75$ ) and for baseline hs-cTn (Peters test, $P=.53$ ). 


\begin{tabular}{|c|c|c|c|c|c|c|c|c|}
\hline Study or Subgroup & $\begin{array}{l}\text { Both Ele } \\
\text { Events }\end{array}$ & $\begin{array}{l}\text { ated } \\
\text { Total }\end{array}$ & $\begin{array}{l}\text { Both Neg } \\
\text { Events }\end{array}$ & $\begin{array}{l}\text { ative } \\
\text { Total }\end{array}$ & Weight & $\begin{array}{l}\text { Odds Ratio } \\
M \cdot \mathrm{H}, \text { Random, } 95 \% \mathrm{Cl}\end{array}$ & $\begin{array}{r}\text { Odds } \\
M-H, \text { Rand }\end{array}$ & $\begin{array}{l}\text { Ratio } \\
\text { dom, } 95 \% \mathrm{Cl}\end{array}$ \\
\hline Aldous 2011 & 29 & 88 & 22 & 189 & $20.7 \%$ & $3.73[1.99,7.00]$ & & $\longrightarrow$ \\
\hline Aldous 2012 & 33 & 185 & 25 & 608 & $23.3 \%$ & $5.06[2.92,8.77]$ & & \\
\hline APACE & 46 & 197 & 33 & 966 & $25.7 \%$ & $8.61[5.33,13.91]$ & & \\
\hline Christ 2010 & 6 & 32 & 0 & 66 & $2.0 \%$ & $32.62[1.77,599.73]$ & & \\
\hline Collinson 2013 & 5 & 47 & 5 & 749 & $8.4 \%$ & $17.71[4.94,63.58]$ & & \\
\hline Eggers 2012 & 14 & 105 & 8 & 200 & $13.9 \%$ & $3.69[1.50,9.12]$ & & \\
\hline Santalo 2013 & 8 & 89 & 2 & 205 & $6.0 \%$ & $10.02[2.08,48.22]$ & & \\
\hline Total $(95 \% \mathrm{Cl})$ & & 743 & & 2983 & $100.0 \%$ & $6.27[4.12,9.54]$ & & \\
\hline Total events & 141 & & 95 & & & & & \\
\hline $\begin{array}{l}\text { Heterogeneity: } \mathrm{Tau}^{2}= \\
\text { Test for overall effect: }\end{array}$ & $\begin{array}{l}.12 ; \mathrm{Chi}^{2} \\
=8.56(P\end{array}$ & $\begin{array}{l}0.34 . \\
.0000\end{array}$ & $\begin{array}{l}\mathrm{df}=6(P= \\
11)\end{array}$ & 1): 1 & $42 \%$ & & $\begin{array}{ll}0.01 & 0.1 \\
\text { Favours both elevated }\end{array}$ & $\begin{array}{rr}1 & 10 \\
\text { Favours bo }\end{array}$ \\
\hline
\end{tabular}

B

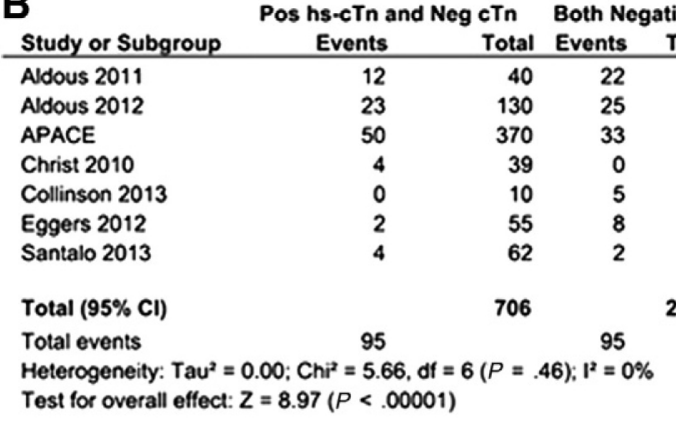

Odds Ratio Odds Ratio

M-H, Random, $95 \% \mathrm{Cl}$ 3.25 [1.45. 7.31]

$5.01[2.74,9.16]$

$4.42[2.80,6.98]$

$16.86[0.88,322.11]$

$6.45[0.33,124.20]$

$0.91[0.19,4.39]$

$7.00[1.25,39.18]$

$4.24[3.09,5.82]$
M-H, Random, $95 \% \mathrm{CI}$

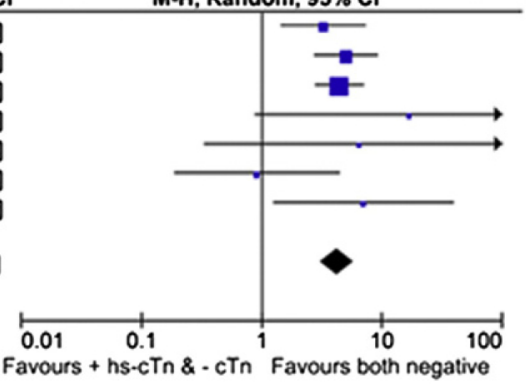

C

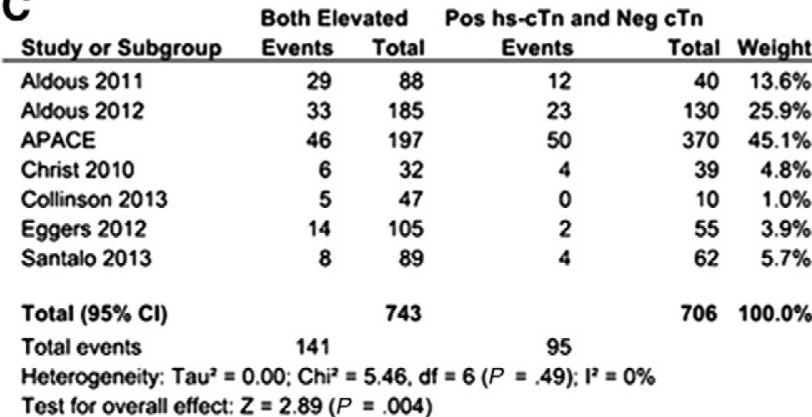

Odds Ratio M-H, Random, 95\% Cl

Forest plots comparing the combination endpoint of death and nonfatal MI during follow-up between patients with elevation of both baseline cTn and baseline hs-cTn and patients with both negative baseline cTn and baseline hs-cTn (A), combination endpoint during follow-up between patients with elevation of baseline hs-cTn and negative baseline cTn and patients with both negative baseline cTn and baseline hs-cTn (B), and combination during follow-up between patients with elevation of both baseline cTn and baseline hs-cTn and patients with elevation of baseline hs-cTn and negative baseline $\mathrm{cTn}(\mathrm{C})$ for patients that presented with chest pain.

\section{Discussion}

This systematic review and collaborative meta-analysis on 8,644 patients demonstrated that hs-cTn and cTn have excellent overall diagnostic accuracy for AMI in patients with chest pain. The hs-cTn assay has the benefit of a significantly greater sensitivity and NPV with a lower negative LR compared with cTn. However, this is at the cost of specificity, PPV, and positive LR. Meta-regression analysis also suggested that time from onset of chest pain to presentation was significantly associated with test performance for baseline $\mathrm{c}$ Tn but was not associated with test performance accuracy for baseline hs-cTn. These data validate previous works suggesting that hs-cTn can more accurately diagnose or exclude AMI early after chest pain. ${ }^{32}$ Prevalence of AMI, STEMI, diabetes mellitus, and male sex also was associated with test performance for baseline cTn but not baseline hs-cTn. When AMI adjudication is performed with hs-cTn as the criterion standard to define AMI, baseline hs-cTn had better test performance as assessed by pooled area under 
the ROC curve compared with baseline cTn. Elevation of baseline hs-cTn identified a greater number of patients who died or had nonfatal MI during follow-up compared with elevation of baseline cTn. Finally, these data demonstrate that baseline elevation of hs-cTn but a negative baseline cTn was associated with an incremental increase in risk for death or nonfatal MI during follow-up. Although troponin assays have previously been compared in meta-analysis, ${ }^{33}$ our metaanalysis is the first to focus specifically on diagnostic and prognostic role of hs-cTn and conventional cTn and performed meta-regression to assess the affect of different variables on diagnostic accuracy. These data support a broader acceptance of hs-cTn.

The development of a universal definition for $\mathrm{AMI}^{34}$ has greatly aided the field of cardiology by providing a means to reliably compare diagnostic tests and therapies. Likewise, establishment of standards for cardiac troponins and adoption of common cut-points ${ }^{30,31,35,36}$ may not only enable improved comparison between assays but also help provide uniform data that physicians can more readily and confidently apply to clinical practice. Adoption of hs-cTn into the ED evaluation of chest pain may significantly alter current practice. Although hs-cTn may enable rapid rule out of patients who present to the ED with chest pain, ${ }^{32,37}$ concern exists that the reduction in PPV and specificity may lead to more extensive cardiovascular testing. Although minimal elevations in hs-cTn may not necessarily identify AMI, it is important to recognize that these patients are at increased risk for adverse outcomes and should receive appropriate medical intervention. ${ }^{4}$ Finally, it is also critical to interpret these biomarkers in the clinical context of the patient. The importance of clinical history and appropriate electrocardiographic evaluation cannot be underestimated. For example, the diagnostic value of a negative troponin is less helpful if the patient's presentation is consistent with unstable angina because the clinical presentation will guide management rather than the biomarker result.

This meta-analysis has several important limitations. To enable appropriate comparison of cTn and hs-cTn in a "realworld" scenario, we excluded studies in which patients were limited to those with a baseline negative troponin because this inherently introduces bias. Similarly, we excluded studies that were limited to only patients with ACS or specific populations. We did not exclude studies with STEMI patients, although this is an electrocardiographic and clinical diagnosis, as we wished to assess the diagnostic accuracy of the assays in all patients with chest pain. The relatively high incidence of $\mathrm{AMI}$ in our population does lead to a bias in the PPV of the test, which is important to acknowledge. However, positive and negative LR should not be influenced by this bias. Other limitations are those inherent to meta-analyses, which include lack of raw or uniform data, and use of different troponin assays and cutpoints. We were also unable to adjust the diagnosis of AMI based on the delta for the rise and/or fall of troponin and the use of longer follow-up may admix events related to ACS with those related to the predictive value of cTn detected in the absence of ACS. Although a random-effect pooling method adjusts for it, another limitation of this meta-analysis is the heterogeneity observed among studies, although this appeared to be low. Finally, meta-regression techniques are limited given the lack of raw patient information and should therefore be viewed with caution and as hypothesis generating.

In conclusion, both cTn and hs-cTn have excellent diagnostic accuracy, but our data support broader use of hs-cTn given the improvements provided in sensitivity, NPV, and identification of patients at risk for adverse outcomes during follow-up.

\section{Conflicts of Interest/Disclosures}

M.J.L., N.C.B., R.O.E., R. Torguson, F.C., S.J.A., S.W.G., K.I., M.S., J.P.C., Y.F., R. Twerenbold, R.W.: none; M.C.: research support and speaker's honoraria from Roche Diagnostics; P.O.C.: consultant for Philips Health Care Incubator and Siemens Point of Care; J.M.: consultant for Philips Health Care Incubator; U.L.: study fees from St Jude Medical and Medtronic, lecture honoraria from St Jude Medical, Medtronic, Sanofi, Aventis, Boehringer Ingelheim, and Bristol:Myers Squibb; C.C.G.: honoraria from Brahms Thermofisher; C. Meune: grant support from Roche Diagnostics and Brahms Thermofisher, and lecture fees from Roche Diagnostics; K.M.E.: honoraria from Siemens Healthcare Diagnostics and consultant for Abbott Laboratories and Fiomi Diagnostics; R.P.: research grant from Abbott Diagnostics; DHS: research grant from Abbott Laboratories and Singulex, Inc; A.H.B.W.: research grant from Singulex, Inc, Roche Laboratories, Alere, and Beckman Coulter, and travel support from Abbott Laboratories; J.O.L.: research support and consultant honoraria from Abbott Diagnostics, Alere, and Roche Diagnostics; A.S.J.: consultant for Roche Laboratories, Radiometer, Abbott Laboratories, Alere Criticical Diagnostics, Ortho Diagnostics, Beckman Coulter, and Amgen; C. Mueller: research support from the European Union, Swiss National Science Foundation, Swiss Heart Foundation, Basel University, University Hospital Basel, Cardiovascular Research Foundation Basel, Stanley Thomas Johnson Foundation, Abbott, ALERE, Beckman Coulter, Brahms, Bühlmann, Critical Diagnostics, Nanosphere, Pronota, Roche, Siemens, and 8sense, and speaker or consulting honoraria from Abbott, ALERE, BG Medicine, Bio Merieux, Brahms, Massachusetts General Hospital, Novartis, Roche, and Siemens.

\section{References}

1. National hospital ambulatory medical care survey: 2010 emergency department summary tables. 20101-33. [www.cdc.gov/nchs/data/ ahcd/nhamcs_emergency/2010_ed_web_tables.pdf]. 
2. Go AS, Mozaffarian D, Roger VL, et al. Heart disease and stroke statistics-2013 update: a report from the American Heart Association. Circulation 2013;127(1):e6-245.

3. Mills NL, Lee KK, McAllister DA, et al. Implications of lowering threshold of plasma troponin concentration in diagnosis of myocardial infarction: cohort study. BMJ 2012;344:e1533.

4. Mills NL, Churchhouse AM, Lee KK, et al. Implementation of a sensitive troponin I assay and risk of recurrent myocardial infarction and death in patients with suspected acute coronary syndrome. JAMA 2011;305(12):1210-6.

5. Body R, Carley S, McDowell G, et al. Rapid exclusion of acute myocardial infarction in patients with undetectable troponin using a high-sensitivity assay. J Am Coll Cardiol 201 1;58(13):1332-9.

6. Stroup DF, Berlin JA, Morton SC, et al. Meta-analysis of observational studies in epidemiology: a proposal for reporting. Meta-analysis of Observational Studies in Epidemiology (MOOSE) group. JAMA 2000;283(15):2008-12.

7. Whiting PF, Rutjes AW, Westwood ME, et al. QUADAS-2: a revised tool for the quality assessment of diagnostic accuracy studies. Ann Intern Med 2012;155(8):529-36.

8. Moses LE, Shapiro D, Littenberg B. Combining independent studies of a diagnostic test into a summary ROC curve: data-analytic approaches and some additional considerations. Stat Med 1993;12 (14): 1293-316.

9. Peters JL, Sutton AJ, Jones DR, et al. Comparison of two methods to detect publication bias in meta-analysis. JAMA 2006;295(6): 676-80.

10. Zamora J, Abraira V, Muriel A, et al. Meta-DiSc: a soffware for metaanalysis of test accuracy data. BMC Med Res Methodol 2006;6:31.

11. Haaf $P$, Reichlin $T$, Twerenbold $R$, et al. Risk stratification in patients with acute chest pain using three high-sensitivity cardiac troponin assays. Eur Heart J 2013;35(6):365-75.

12. Aldous SJ, Florkowski CM, Crozier IG, et al. Comparison of high sensitivity and contemporary troponin assays for the early detection of acute myocardial infarction in the emergency department. Ann Clin Biochem 2011;48:241-8.

13. Aldous SJ, Florkowski CM, Crozier IG, et al. High sensitivity troponin outperforms contemporary assays in predicting major adverse cardiac events up to two years in patients with chest pain. Ann Clin Biochem 2011;48:249-55.

14. Aldous SJ, Florkowski CM, Crozier IG, et al. The performance of high sensitivity troponin for the diagnosis of acute myocardial infarction is underestimated. Clin Chem Lab Med 2012;50(4):727-9.

15. Aldous SJ, Richards $M$, Cullen $L$, et al. Diagnostic and prognostic utility of early measurement with high-sensitivity troponin T assay in patients presenting with chest pain. CMAJ 2012;184(5):E260-8.

16. Christ $M$, Popp S, Pohlmann H, et al. Implementation of high sensitivity cardiac troponin T measurement in the emergency department. Am J Med 2010;123(12):1134-42.

17. Collinson PO, Gaze DC, Thokala P, et al. Randomised Assessment of Treatment using Panel Assay of Cardiac markers-Contemporary Biomarker Evaluation (RATPAC CBE). Health Technol Assess 2013;17 (15):1-122. [v-vi].

18. Eggers $K M$, Venge $P$, Lindahl B. High-sensitive cardiac troponin $T$ outperforms novel diagnostic biomarkers in patients with acute chest pain. Clin Chim Acta 2012;413(13-14):1135-40.

19. Freund $Y$, Chenevier-Gobeaux $C$, Bonnet $P$, et al. High-sensitivity versus conventional troponin in the emergency department for the diagnosis of acute myocardial infarction. Crit Care 2011;15(3): R147.
20. Hammerer-Lercher A, Ploner T, Neururer S, et al. High-sensitivity cardiac troponin T compared with standard troponin T testing on emergency department admission: how much does it add in everyday clinical practice? J Am Heart Assoc 2013;2(3):e000204.

21. Inoue K, Suwa S, Ohta H, et al. Heart fatty acid-binding protein offers similar diagnostic performance to high-sensitivity troponin $\mathrm{T}$ in emergency room patients presenting with chest pain. Circ J 201 1;75 (12):2813-20.

22. Keller T, Zeller T, Peetz D, et al. Sensitive troponin I assay in early diagnosis of acute myocardial infarction. N Engl J Med 2009;361(9): 868-77.

23. Lotze $\mathrm{U}$, Lemm $\mathrm{H}$, Heyer $\mathrm{A}$, et al. Combined determination of highly sensitive troponin $T$ and copeptin for early exclusion of acute myocardial infarction: first experience in an emergency department of a general hospital. Vasc Health Risk Manag 201 1;7:509-15.

24. Melki D, Lind S, Agewall S, et al. Diagnostic value of high sensitive troponin $\mathrm{T}$ in chest pain patients with no persistent ST-elevations. Scand Cardiovasc J 201 1;45(4):198-204.

25. Meune C, Zuily S, Wahbi K, et al. Combination of copeptin and highsensitivity cardiac troponin T assay in unstable angina and non-STsegment elevation myocardial infarction: a pilot study. Arch Cardiovasc Dis 2011;104(1):4-10.

26. Pracon R, Kruk M, Jakubczak $B$, et al. Superior early diagnostic performance of a sensitive cardiac troponin assay as compared to a standard troponin test in the diagnosis of acute myocardial infarction. Kardiol Pol 2012;70(2):131-8.

27. Santalo M, Martin A, Velilla J, et al. Using high-sensitivity troponin T: the importance of the proper gold standard. Am J Med 2013;126(8): 709-17.

28. Schreiber DH, Agbo C, Wu AH. Short-term (90 min) diagnostic performance for acute non-ST segment elevation myocardial infarction and 30-day prognostic evaluation of a novel thirdgeneration high sensitivity troponin I assay. Clin Biochem 2012;45 (16-17):1295-301.

29. Sebbane M, Lefebvre S, Kuster N, et al. Early rule out of acute myocardial infarction in ED patients: value of combined highsensitivity cardiac troponin T and ultrasensitive copeptin assays at admission. Am J Emerg Med 2013;31(9):1302-8.

30. Troponin assay analytical characteristics. International Federation of Clinical Chemistry and Laboratory Medicine. 2013.

31. Jaffe AS, Ordonez-Llanos J. High-sensitivity cardiac troponin: from theory to clinical practice. Rev Esp Cardiol 2013;66(9):687-91.

32. Cullen L, Mueller C, Parsonage WA, et al. Validation of highsensitivity troponin I in a 2-hour diagnostic strategy to assess 30-day outcomes in emergency department patients with possible acute coronary syndrome. J Am Coll Cardiol 2013;62(14):1242-9.

33. Goodacre S, Thokala P, Carroll C, et al. Systematic review, metaanalysis and economic modelling of diagnostic strategies for suspected acute coronary syndrome. Health Technol Assess 2013;17 (1):1-188. [v-vi].

34. Thygesen K, Alpert JS, White HD. Universal definition of myocardial infarction. Eur Heart J 2007;28(20):2525-38.

35. Apple FS, Collinson PO. Analytical characteristics of high-sensitivity cardiac troponin assays. Clin Chem 2012;58(1):54-61.

36. Thygesen K, Mair J, Giannitsis E, et al. How to use high-sensitivity cardiac troponins in acute cardiac care. Eur Heart J 2012;33(18): 2252-7.

37. Reichlin T, Schindler C, Drexler B, et al. One-hour rule-out and rule-in of acute myocardial infarction using high-sensitivity cardiac troponin T. Arch Intern Med 2012;172(16):1211-8. 


\section{Appendix}

Supplementary Table I. Appraisal of included studies

\begin{tabular}{|c|c|c|c|c|c|c|c|}
\hline Study & $\begin{array}{l}\text { Standard } \\
\text { troponin } \\
\text { assays }\end{array}$ & $\begin{array}{l}\text { Prespecified } \\
\text { cut-points }\end{array}$ & $\begin{array}{l}\text { Study } \\
\text { design }\end{array}$ & $\begin{array}{c}\text { Consecutive } \\
\text { patient } \\
\text { inclusion }\end{array}$ & $\begin{array}{l}\text { Withdrawals } \\
\text { reported }\end{array}$ & AMI definition & $\begin{array}{l}\text { Troponin } \\
\text { used to } \\
\text { define AMI }\end{array}$ \\
\hline $\begin{array}{l}\text { Aldous } \\
\text { et al } 2012\end{array}$ & Yes & Yes & Prospective & No & Yes & $\begin{array}{l}\text { Universal definition with physician } \\
\text { adjudication. Biomarker elevation with a } \\
\text { rise or fall or signs of } C A D\end{array}$ & Conventional \\
\hline $\begin{array}{l}\text { Aldous } \\
\text { et al } 2011\end{array}$ & Yes & Yes & Prospective & No & Yes & $\begin{array}{l}\text { Universal definition with physician } \\
\text { adjudication. Biomarker elevation with } \\
\text { a } 20 \% \text { rise or fall or signs of CAD }\end{array}$ & Conventional \\
\hline APACE & Yes & Yes & Prospective & Yes & Yes & $\begin{array}{l}\text { Universal definition with physician } \\
\text { adjudication. Biomarker elevation with } \\
\text { a } 30 \% \text { rise or fall or signs CAD }\end{array}$ & Conventional \\
\hline Christ et al & Yes & Yes & Retrospective & Yes & Yes & $\begin{array}{l}\text { Universal definition with physician } \\
\text { adjudication. Biomarker elevation with } \\
\text { a } 20 \% \text { rise or fall or signs of } C A D\end{array}$ & Conventional \\
\hline Collinson et al & Yes & Yes & Prospective & No & Yes & $\begin{array}{l}\text { Universal definition with physician } \\
\text { adjudication. Biomarker elevation with } \\
\text { a rise or fall or signs of CAD }\end{array}$ & Conventional \\
\hline Eggers et al & Yes & Yes & Prospective & Yes & No & $\begin{array}{l}\text { Universal definition with physician } \\
\text { adjudication Biomarker elevation with } \\
\text { a } 20 \% \text { rise or fall, an absolute change } \\
\text { of } \geq 5 \mathrm{ng} / \mathrm{L} \text {, or signs of CAD }\end{array}$ & Conventional \\
\hline Freund et al & Yes & Yes & Prospective & Yes & Yes & $\begin{array}{l}\text { Universal definition with physician } \\
\text { adjudication. Biomarker elevation } \\
\text { with symptoms or signs of CAD }\end{array}$ & Conventional \\
\hline $\begin{array}{l}\text { Hammerer- } \\
\text { Lercher et al }\end{array}$ & Yes & Yes & Retrospective & Yes & Yes & $\begin{array}{l}\text { Universal definition with physician } \\
\text { adjudication. Biomarker elevation with } \\
\text { a rise or fall or signs of } C A D\end{array}$ & Conventional \\
\hline Inoue et al & Yes & Yes & Prospective & Yes & Yes & $\begin{array}{l}\text { Universal definition with physician } \\
\text { adjudication. }\end{array}$ & Conventional \\
\hline Keller et al & Yes & Yes & Prospective & Yes & Yes & $\begin{array}{l}\text { Universal definition with physician } \\
\text { adjudication. Biomarker elevation with } \\
\text { a } 20 \% \text { rise or fall or signs of } C A D\end{array}$ & Conventional \\
\hline Lotze et al & Yes & Yes & Prospective & Yes & Yes & $\begin{array}{l}\text { Universal definition with physician } \\
\text { adjudication. Biomarker elevation with } \\
\text { a rise or fall or signs of CAD }\end{array}$ & Combination \\
\hline Melki et al & Yes & Yes & Prospective & No & Yes & $\begin{array}{l}\text { Universal definition with physician } \\
\text { adjudication. Biomarker elevation with } \\
\text { a rise or fall or signs of CAD }\end{array}$ & Conventional \\
\hline Meune et al & Yes & Yes & Prospective & Yes & Yes & $\begin{array}{l}\text { Universal definition with physician } \\
\text { adjudication. Biomarker elevation with } \\
\text { a rise or fall }\end{array}$ & Conventional \\
\hline Pracon et al & Yes & Yes & Prospective & Yes & Yes & $\begin{array}{l}\text { Universal definition with physician } \\
\text { adjudication. Biomarker elevation with } \\
\text { a rise or fall or signs of CAD }\end{array}$ & Combination \\
\hline Santalo et al & Yes & Yes & Prospective & Yes & Yes & $\begin{array}{l}\text { Universal definition with physician } \\
\text { adjudication. Biomarker elevation with } \\
\text { a } 20 \% \text { rise or fall }\end{array}$ & Conventional \\
\hline Schreiber et al & Yes & Yes & Prospective & No & Yes & $\begin{array}{l}\text { Universal definition with physician } \\
\text { adjudication. Biomarker elevation with } \\
\text { a rise or fall }\end{array}$ & Combination \\
\hline Sebbane et al & Yes & Yes & Prospective & Yes & Yes & $\begin{array}{l}\text { Universal definition with physician } \\
\text { adjudication. Biomarker elevation with } \\
\text { a rise or fall or signs of CAD }\end{array}$ & Conventional \\
\hline
\end{tabular}


Supplementary Table II. Number of TPs, FPs, FNs, and TNs based on the baseline cTn at presentation or baseline hs-cTn at presentation cutpoint and whether the patient experienced AMI

cTn

\section{Study Conventional cTn cut-point}

TP (n) FP (n) FN (n) TN (n) Sensitivity

$(\%)$

$\begin{array}{rrrrr}175 & 26 & 30 & 708 & 85.4 \\ 82 & 21 & 28 & 201 & 74.5 \\ 168 & 29 & 66 & 1270 & 71.8 \\ 13 & 11 & 7 & 106 & 65.0 \\ 53 & 29 & 10 & 739 & 84.1 \\ 92 & 13 & 36 & 219 & 71.9 \\ 32 & 9 & 13 & 263 & 71.1 \\ & & & & \\ 35 & 48 & 5 & 352 & 87.5 \\ & & & & \\ 98 & 33 & 65 & 87 & 60.1 \\ 300 & 83 & 113 & 1322 & 72.6 \\ 11 & 38 & 2 & 91 & 84.6 \\ 90 & 7 & 24 & 112 & 79.0 \\ 12 & 7 & 1 & 38 & 92.3 \\ 69 & 20 & 15 & 83 & 82.1 \\ 61 & 28 & 17 & 250 & 78.2 \\ 9 & 14 & 3 & 439 & 75.0 \\ 38 & 10 & 13 & 133 & 74.5\end{array}$

Specificity

(\%)

96.5

90.5

79.6

85.3

54.2

64.6

87.6

78.0

42.2

88.0

74.8

78.3

22.4

92.8

63.2

77.5

68.5

39.1

79.2
NPV

(\%)

hs-cTn

\begin{tabular}{|c|c|c|c|c|c|c|c|c|c|}
\hline Study & hs-cTn cut-point & $\operatorname{TP}(n)$ & $\mathrm{FP}(\mathrm{n})$ & $\mathrm{FN}(\mathrm{n})$ & $\mathrm{TN}(\mathrm{n})$ & Sensitivity (\%) & Specificity (\%) & PPV (\%) & NPV (\%) \\
\hline $\begin{array}{l}\text { Aldous } \\
\text { et al } 2012\end{array}$ & Roche HS TnT, 14 ng/L & 181 & 134 & 24 & 600 & 88.3 & 81.7 & 57.5 & 96.2 \\
\hline $\begin{array}{l}\text { Aldous } \\
\text { et al } 2011\end{array}$ & Roche HS TnT, 14 ng/L & 92 & 36 & 18 & 186 & 83.6 & 83.8 & 71.9 & 91.2 \\
\hline APACE & Roche HS TnT, $14 \mathrm{ng} / \mathrm{L}$ & 221 & 346 & 13 & 953 & 94.4 & 73.4 & 39.0 & 98.7 \\
\hline Christ et al & Roche HS TnT, $14 \mathrm{ng} / \mathrm{L}$ & 19 & 45 & 1 & 72 & 95.0 & 61.5 & 29.7 & 98.6 \\
\hline Collinson et al & Beckman AccuTnl, $40 \mathrm{ng} / \mathrm{L}$ & 43 & 15 & 20 & 757 & 68.2 & 98.1 & 74.1 & 97.4 \\
\hline Eggers et al & Roche HS TnT, $14 \mathrm{ng} / \mathrm{L}$ & 101 & 59 & 27 & 173 & 78.9 & 74.6 & 63.1 & 86.5 \\
\hline Freund et al & Roche HS TnT, $14 \mathrm{ng} / \mathrm{L}$ & 42 & 48 & 3 & 224 & 93.3 & 82.4 & 46.7 & 98.7 \\
\hline $\begin{array}{l}\text { Hammerer- } \\
\text { Lercher et al }\end{array}$ & Roche HS TnT, 14 ng/L & 36 & 80 & 4 & 320 & 90.0 & 80.0 & 31.0 & 98.8 \\
\hline Inoue et al & Roche HS TnT, $14 \mathrm{ng} / \mathrm{L}$ & 141 & 59 & 22 & 61 & 86.5 & 50.8 & 70.5 & 73.5 \\
\hline Keller et al & Siemens sensitive Tnl Ultra, 40 ng/L & 375 & 138 & 38 & 1267 & 90.8 & 90.2 & 73.1 & 97.1 \\
\hline Lotze et al & Roche HS TnT, 14 ng/L & 12 & 60 & 1 & 69 & 92.3 & 53.5 & 16.7 & 98.6 \\
\hline Melki et al & Roche HS TnT, 14 ng/L & 111 & 31 & 3 & 88 & 97.4 & 73.9 & 78.2 & 96.7 \\
\hline Meune et al & Roche HS TnT, 14 ng/L & 12 & 12 & 1 & 33 & 92.3 & 73.3 & 50.0 & 97.1 \\
\hline Pracon et al & Abbott Architect Stat Tnl, 28 ng/L & 73 & 12 & 11 & 91 & 86.9 & 88.3 & 85.9 & 89.2 \\
\hline Santalo et al & Roche HS TnT, $14 \mathrm{ng} / \mathrm{L}$ & 70 & 79 & 8 & 199 & 89.7 & 71.6 & 47.0 & 96.1 \\
\hline Schreiber et al & Singulex Erenna HS-Tnl, 8 ng/L & 10 & 80 & 2 & 373 & 83.3 & 82.3 & 11.1 & 99.5 \\
\hline Sebbane et al & Roche HS TnT, $14 \mathrm{ng} / \mathrm{L}$ & 39 & 22 & 12 & 121 & 76.5 & 84.6 & 63.9 & 91.0 \\
\hline
\end{tabular}


Supplementary Table III. Number of TPs, FPs, FNs, and TNs based on the second cTh or second hs-cTn cutpoint and whether the patient experienced AMI for studies providing this data

\section{Study \\ Time since presentation (h) TP (n) FP (n) FN (n) TN (n) Sensitivity (\%) Specificity (\%) \\ PPV (\%) NPV (\%)}

cTn at second serial blood sampling

Aldous et al 2012

Aldous et al 2011

APACE

Christ et al

Collinson et al

Freund et al

Meune et al

Pracon et al

Santalo et al

Schreiber et al
hs-cTn at second serial blood sampling

Aldous et al 2012

Aldous et al 2011

APACE

Christ et al

Collinson et al

Freund et al

Meune et al

Pracon et al

Santalo et al

Schreiber et al

2
6
2
6
1.5
6
3
4
2
1.5

2

6

2

4

1.5

6

3

3

2

1.5

$\begin{array}{rrrr}189 & 30 & 16 & 704 \\ 100 & 26 & 10 & 196 \\ 88 & 23 & 11 & 788 \\ 13 & 12 & 7 & 105 \\ 13 & 12 & 1 & 643 \\ 9 & 2 & 1 & 107 \\ 11 & 9 & 2 & 36 \\ 20 & 8 & 2 & 22 \\ 63 & 28 & 8 & 239 \\ 7 & 14 & 2 & 384\end{array}$

92.2

90.9

88.9

65.0

92.9

90.0

84.6

90.9

88.7

77.8

$\begin{array}{rrrr}189 & 149 & 16 & 585 \\ 100 & 41 & 10 & 181 \\ 96 & 231 & 2 & 579 \\ 15 & 41 & 5 & 76 \\ 9 & 7 & 2 & 647 \\ 5 & 12 & 0 & 44 \\ 13 & 14 & 0 & 31 \\ 11 & 2 & 1 & 16 \\ 65 & 75 & 4 & 213 \\ 9 & 72 & 0 & 326\end{array}$

92.2

90.9

98.0

75.0

81.8

100.0

100.0

91.7

94.2

100.0
95.9

88.3

97.2

89.7

98.2

98.2

80.0

73.3

89.5

96.5

79.7

81.5

71.5

65.0

98.9

78.6

68.9

88.9

74.0

81.9
86.3

79.4

79.3

52.0

52.0

81.8

55.0

71.4

69.2

33.3

55.9

97.3

$70.9 \quad 94.8$

$29.4 \quad 99.7$

$26.8 \quad 93.8$

$56.3 \quad 99.7$

29.4

48.1

100.0

100.0

84.6

94.1

46.4

98.2

11.1

100.0

Abbreviations: $T P$, True positive; $F P$, false positive; $F N$, false negative; $T N$, true negative.

Supplementary Table IV. Number of TPs, FPs, FNs, and TNs based on the baseline cTn or baseline hs-cTn cut-point and whether the patient experienced AMI when AMI was defined using the cut-point for the hs-cTn assay

\begin{tabular}{|c|c|c|c|c|c|c|c|c|}
\hline Study & $\operatorname{TP}(n)$ & $\mathbf{F P}(\mathbf{n})$ & FN (n) & TN (n) & Sensitivity (\%) & Specificity (\%) & PPV (\%) & NPV (\%) \\
\hline \multicolumn{9}{|l|}{ Baseline cTn } \\
\hline Aldous et al 2011 & 105 & 26 & 24 & 177 & 81.4 & 87.2 & 80.2 & 88.1 \\
\hline APACE & 163 & 20 & 149 & 1201 & 52.2 & 98.4 & 89.1 & 89.0 \\
\hline Christ et al & 13 & 11 & 22 & 91 & 37.1 & 89.2 & 54.2 & 80.5 \\
\hline Collinson et al & 54 & 28 & 12 & 737 & 81.8 & 96.3 & 65.9 & 98.4 \\
\hline Melki et al & 92 & 9 & 39 & 93 & 70.2 & 91.2 & 91.1 & 70.5 \\
\hline Santalo et al & 89 & 62 & 13 & 192 & 87.3 & 75.6 & 58.9 & 93.7 \\
\hline \multicolumn{9}{|l|}{ Baseline hs-cTn } \\
\hline Aldous et al 2011 & 116 & 14 & 13 & 189 & 89.9 & 93.1 & 89.2 & 93.6 \\
\hline APACE & 281 & 286 & 31 & 935 & 90.1 & 76.6 & 49.6 & 96.8 \\
\hline Christ et al & 33 & 31 & 2 & 71 & 94.3 & 69.6 & 51.6 & 97.3 \\
\hline Collinson et al & 45 & 13 & 21 & 756 & 68.2 & 98.3 & 77.6 & 97.3 \\
\hline Melki et al & 128 & 18 & 3 & 84 & 97.7 & 82.4 & 87.7 & 96.6 \\
\hline Santalo et al & 61 & 25 & 41 & 237 & 59.8 & 90.5 & 70.9 & 85.3 \\
\hline
\end{tabular}

Abbreviations: $T P$, true positive; FP, false positive; FN, false negative; $T N$, true negative. 
Supplementary Table V. Area under the ROC curves for the admission and second conventional and hs-cTn for the diagnosis of AMI

\begin{tabular}{|c|c|c|c|c|c|}
\hline Study & $\begin{array}{c}\text { Conventional cTn, } \\
\text { AUC } \pm \text { SE }\end{array}$ & $\begin{array}{l}\text { Hs-cTn, } \\
\text { AUC } \pm \text { SE }\end{array}$ & $\begin{array}{l}\text { Time to next } \\
\text { troponin }(\mathrm{h})\end{array}$ & $\begin{array}{l}\text { Next conventional cTn, } \\
\text { AUC } \pm \text { SE }\end{array}$ & $\begin{array}{c}\text { Next Hs-cTn, } \\
\text { AUC } \pm \text { SE }\end{array}$ \\
\hline Aldous et al 2012 & $0.96 \pm 0.01$ & $0.92 \pm 0.01$ & 2 & $0.98 \pm 0.01$ & $0.93 \pm 0.01$ \\
\hline Aldous et al 2011 & $0.88 \pm 0.02$ & $0.90 \pm 0.02$ & 6 & $0.93 \pm 0.02$ & $0.94 \pm 0.02$ \\
\hline APACE & $0.79 \pm 0.06$ & $0.92 \pm 0.02$ & 2 & $0.97 \pm 0.02$ & $0.97 \pm 0.01$ \\
\hline Christ et al & $0.89 \pm 0.04$ & $0.91 \pm 0.03$ & 6 & $0.97 \pm 0.02$ & $0.97 \pm 0.01$ \\
\hline Collinson et al & $0.94 \pm 0.02$ & $0.92 \pm 0.02$ & 1.5 & $0.95 \pm 0.05$ & $0.94 \pm 0.06$ \\
\hline Eggers et al & $0.91 \pm 0.02$ & $0.85 \pm 0.02$ & & NR & NR \\
\hline Freund et al & $0.93 \pm 0.02$ & $0.93 \pm 0.02$ & 6 & $0.85 \pm 0.10$ & $0.94 \pm 0.05$ \\
\hline Hammerer-Lercher ef al & $0.91 \pm 0.02$ & $0.94 \pm 0.01$ & & NR & NR \\
\hline Inove et al & $0.68 \pm 0.03$ & $0.73 \pm 0.03$ & & NR & NR \\
\hline Keller et al & $0.85 \pm 0.02$ & $0.96 \pm 0.02$ & 3 & $0.98 \pm 0.01$ & $0.98 \pm 0.01$ \\
\hline Lotze ef al & $0.85 \pm 0.03$ & $0.87 \pm 0.03$ & & NR & NR \\
\hline Melki et al & $0.93 \pm 0.02$ & $0.95 \pm 0.02$ & 2 & $0.96 \pm 0.01$ & $0.96 \pm 0.01$ \\
\hline Meune et al & $0.95 \pm 0.05$ & $0.92 \pm 0.04$ & 3 & $0.98 \pm 0.02$ & $0.97 \pm 0.02$ \\
\hline Pracon et al & $0.86 \pm 0.03$ & $0.92 \pm 0.02$ & 4 & $0.86 \pm 0.05$ & $0.91 \pm 0.06$ \\
\hline Santalo et al & $0.83 \pm 0.12$ & $0.81 \pm 0.10$ & 2 & $0.96 \pm 0.04$ & $0.84 \pm 0.09$ \\
\hline Schreiber et al & $0.90 \pm 0.01$ & $0.94 \pm 0.01$ & 1.5 & $0.87 \pm 0.02$ & $0.98 \pm 0.01$ \\
\hline Sebbane et al & $0.90 \pm 0.03$ & $0.89 \pm 0.02$ & & NR & NR \\
\hline
\end{tabular}

Abbreviations: AUC, Area under the ROC curve; NR, not reported. 


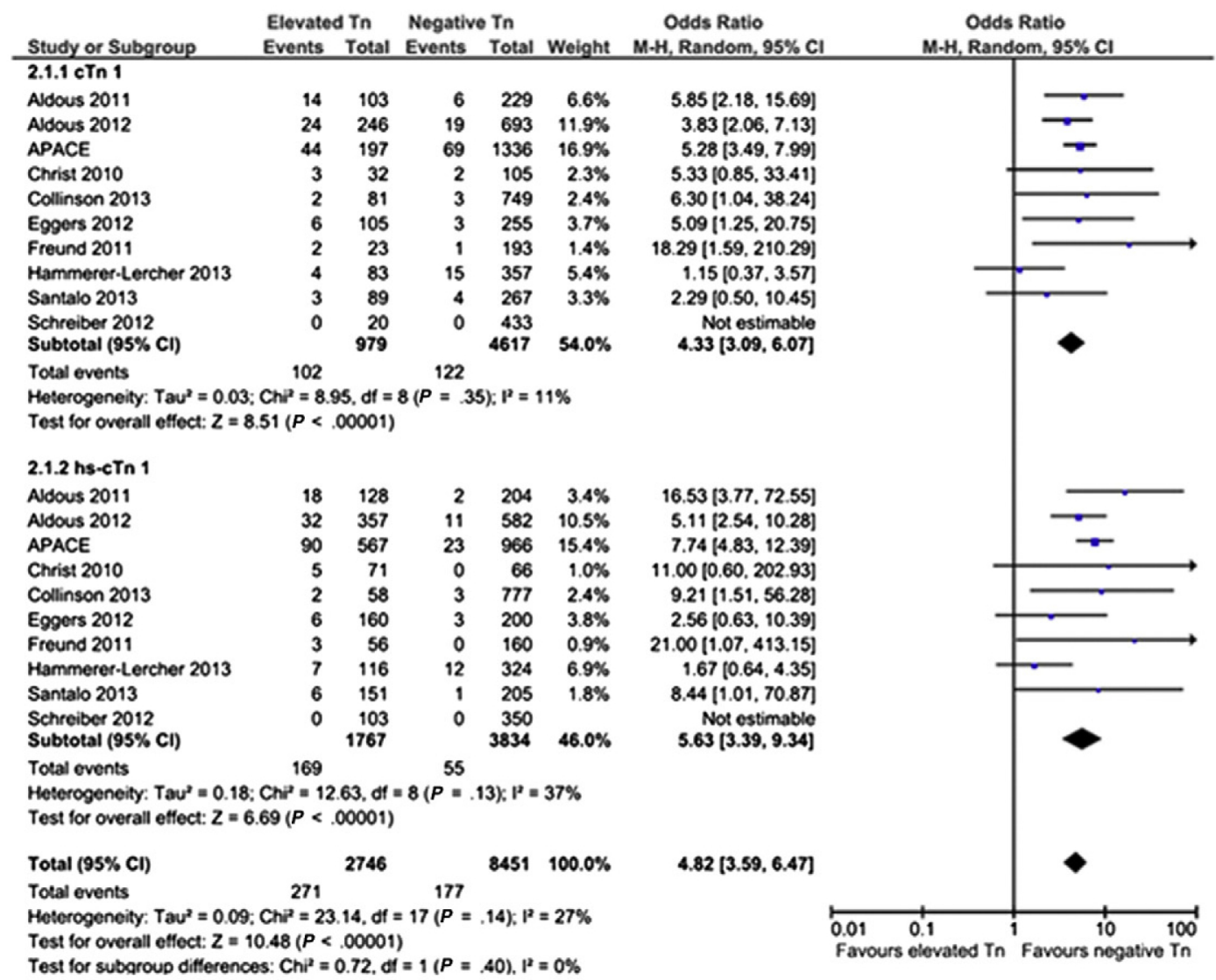

Elevated Tn Negative Tn

Odds Ratio

Study or Subgroup Events Total Events Total Woight M-H, Random, 95\% CI 2.2.1 cTn 1

$\begin{array}{lrrrrr}\text { Aldous 2011 } & 23 & 103 & 31 & 229 & 12.0 \% \\ \text { Addous 2012 } & 32 & 246 & 24 & 693 & 13.5 \% \\ \text { APACE } & 19 & 197 & 60 & 1336 & 13.9 \% \\ \text { Christ 2010 } & 3 & 32 & 3 & 105 & 2.1 \% \\ \text { Collinson 2013 } & 3 & 81 & 2 & 749 & 1.7 \% \\ \text { Eggers 2012 } & 10 & 105 & 7 & 255 & 5.2 \% \\ \text { Santalo 2013 } & 5 & 89 & 2 & 267 & 2.0 \% \\ \text { Subtotal (95\% CI) } & & 853 & & 3634 & 50.3 \% \\ \text { Total events } & 95 & & 129 & & \end{array}$

Heterogeneity: Tau $^{2}=0.10 ;$ Chi $^{2}=9.39, \mathrm{df}=6(P=.15) ; \mathrm{I}^{2}=36 \%$

Test for overall effect: $Z=5.52(P<.00001)$

\subsection{2 hs-cTn 1}

Aldous 2011

Aldous 2012

APACE

Christ 2010

Collinson 2013

Eggers 2012

Santalo 2013

Subtotal $(95 \% \mathrm{Cl})$

Total events

Heterogeneity: Tau $^{2}=0.00 ; \mathrm{Chi}^{2}=5.83 . \mathrm{df}=6(P=.44) ; \mathrm{P}^{2}=0 \%$

Test for overall effect: $Z=8.38(P<.00001)$

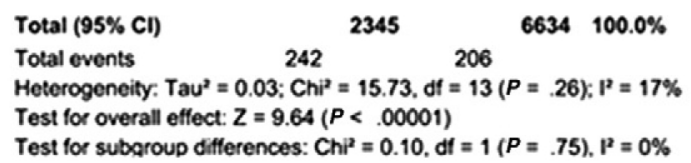

$1.84[1.01,3.34]$

4.17 [2.40, 7.23]

2.27 [1.32, 3.89]

$3.52[0.67,18.36]$

14.37 [2.36, 87.28]

3.73 [1.38, 10.08]

$7.89[1.50,41.40]$

$3.18[2.11,4.80]$
Odds Ratio

M.H, Random, $95 \% \mathrm{Cl}$ 


\section{Supplementary Figure 1}

\begin{tabular}{|c|c|c|c|c|c|c|c|c|}
\hline Study or Subgroup & \multicolumn{2}{|c|}{ Elevated Tn } & \multicolumn{2}{|c|}{ Negative Tn } & \multirow[t]{2}{*}{ Weight } & \multirow[t]{2}{*}{$\begin{array}{l}\text { Odds Ratio } \\
\text { M.H, Random, } 95 \% \text { CI }\end{array}$} & \multicolumn{2}{|c|}{$\begin{array}{l}\text { Odds Ratio } \\
\text { M.H, Random, } 95 \% \mathrm{Cl}\end{array}$} \\
\hline \multicolumn{7}{|l|}{$2.3 .1 \mathrm{cTn} 1$} & & \\
\hline APACE & 46 & 197 & 83 & 1336 & $34.8 \%$ & $4.60[3.09 .6 .85]$ & & \\
\hline Christ 2010 & 6 & 32 & 4 & 105 & $3.1 \%$ & $5.83[1,53,22.18]$ & & \\
\hline Collinson 2013 & 5 & 81 & 5 & 749 & $3.5 \%$ & $9.79[2.77,34.58]$ & & \\
\hline Eggers 2012 & 14 & 105 & 10 & 255 & $7.7 \%$ & $3.77[1.62,8.79]$ & & \\
\hline $\begin{array}{l}\text { Santalo } 2013 \\
\text { Subtotal }(95 \% \mathrm{CI})\end{array}$ & 8 & $\begin{array}{r}89 \\
504\end{array}$ & 6 & $\begin{array}{r}267 \\
2712\end{array}$ & $\begin{array}{r}4.7 \% \\
53.7 \%\end{array}$ & $\begin{array}{r}4.30[1.45,12.75] \\
4.73[3.43,6.51]\end{array}$ & & \\
\hline Total events & 79 & & 108 & & & & & \\
\hline \multicolumn{9}{|c|}{$\begin{array}{l}\text { Heterogeneity: Tau }=0.00 ; \mathrm{Chi}^{2}=1.70 . \mathrm{df}=4(P=.79) ; \mathrm{P}^{2}=0 \% \\
\text { Test for overall effect: } Z=9.51(P<.00001)\end{array}$} \\
\hline \multicolumn{9}{|l|}{ 2.3.2 hs-cTn 1} \\
\hline APACE & 96 & 567 & 33 & 966 & $32.6 \%$ & $5.76[3.82 .8 .69]$ & & \\
\hline Christ 2010 & 10 & 71 & 0 & 66 & $0.7 \%$ & 22.71 [1.30. 395.77] & & \\
\hline Collinson 2013 & 5 & 58 & 5 & 777 & $3.4 \%$ & $14.57[4.09 .51 .89]$ & & \\
\hline Eggers 2012 & 16 & 160 & 8 & 200 & $7.2 \%$ & $2.67[1.11,6.40]$ & & \\
\hline $\begin{array}{l}\text { Santalo } 2013 \\
\text { Subtotal }(95 \% \mathrm{CI})\end{array}$ & 12 & $\begin{array}{r}151 \\
1007\end{array}$ & 2 & $\begin{array}{r}205 \\
2214\end{array}$ & $\begin{array}{r}2.4 \% \\
46.3 \%\end{array}$ & $\begin{array}{l}8.76[1,93,39.76] \\
6.05[3.42,10.71]\end{array}$ & & \\
\hline Total ovents & 139 & & 48 & & & & & \\
\hline \multicolumn{9}{|c|}{$\begin{array}{l}\text { Heterogeneity: Tau }=0.14 ; \mathrm{Chi}^{2}=6.20, \mathrm{df}=4(P=.18) ; \mathrm{P}^{2}=35 \% \\
\text { Test for overall effect: } Z=6.18(P<.00001)\end{array}$} \\
\hline Total (95\% CI) & & 1511 & & 4926 & $100.0 \%$ & $5.16[4,08,6.52]$ & & \\
\hline Total events & 218 & & 156 & & & & & \\
\hline $\begin{array}{l}\text { Heterogeneity: Tauz } \\
\text { Test for overall effect }\end{array}$ & $\begin{array}{l}0.00 ; \mathrm{Chi}^{2} \\
Z=13.70\end{array}$ & $\begin{array}{l}=8.51 \\
P<.0 \\
P<.5\end{array}$ & $\begin{array}{l}d f=9(P \\
0001)\end{array}$ & (48); & $\mathrm{P}=0 \%$ & & $\begin{array}{lc}0.01 & 0.1 \\
\text { Favours elevated in }\end{array}$ & Favours nes \\
\hline
\end{tabular}

(Continued.) 